\title{
RESIDUAL-BASED A POSTERIORI ERROR ESTIMATES FOR A CONFORMING FINITE ELEMENT DISCRETIZATION OF THE STOKES-DARCY COUPLED PROBLEM
}

\section{HOUEDANOU KOFFI WILFRID}

Département de Mathématiques

Université d'Abomey-Calavi (UAC)

République du Bénin

e-mail: khouedanou@yahoo.fr

\begin{abstract}
We consider in this paper, a new a posteriori residual type error estimators for the Stokes-Darcy coupled problem analyzed in [1] on isotropic meshes. Our analysis covers two-and three-dimensional domains, conforming discretizations as well as different elements. We derive a reliable and efficient residual-based a posteriori error estimator for this coupled problem. The proof of reliability makes use of suitable auxiliary problems, continuous inf-sup conditions satisfied by the bilinear forms involved, and local approximation properties. The a posteriori error estimate is based on a suitable evaluation on the residual of the finite element solution. It is proven that the a posteriori error estimate provided in this paper is both reliable and efficient.
\end{abstract}

2010 Mathematics Subject Classification: 74S05, 74S10, 74S15, 74S20, 74S25, 74S30.

Keywords and phrases: mixed finite elements, Stokes and Darcy equations, a posteriori error analysis.

Received August 9, 2019

(ㄷ) 2019 Scientific Advances Publishers 


\section{Introduction}

The coupling of Stokes and Darcy flow problems has received significant attention over the past several years due to its importance in modelling problems such as surface fluid flow coupled with flow in porous media, see [6]-[9] and the references therein. Mathematical justification for the interface boundary condition was derived in Jäger and Mikelic [7] and Mardal et al. [8] for the robust finite element constructions. Wellposedness and convergence of the finite element method can be found in [1]. A posteriori error estimates are computable quantities in terms of the discrete solution of data that measure the actual discrete errors without the knowledge of exact solutions. They are essential for designing algorithms with adaptive mesh refinement which equidistribute the computational effort and optimize the approximation efficiency. It ensures a higher density of nodes in a certain area of the given domain, where the solution is more difficult to be approximated, using an a posteriori error indicator. Ever since the pioneering work of Bieterman and Babuska [10], the adaptive finite element method based on a posteriori error estimates has been extensively investigated. In [4], two a posteriori error estimators for the mini-element discretization of the Stokes equations were presented. Recently, a residual-based a posteriori error estimator for the Stokes-Darcy coupled problem was presented in [11], where Bernardi-Raugel and Raviart-Thomas elements for the velocity and piecewise constants for the pressures were considered. A posteriori error estimates for the finite element approximation of the distributed optimal control problems governed by the Stokes equations was derived in [12, 35, 36].

The purpose of this work is to derive a reliable and efficient residualbased a posteriori error estimator for the Stokes-Darcy coupled problem analyzed in [1]. Though one might think a priori that this should follow simply by combining the corresponding approaches already available for the Stokes and Darcy problems, the analysis below will show that this idea works only partially since further difficulties and several technical issues arise along the way. In this respect, it is important to remark that, 
on one hand, the transmission conditions stop us from splitting the analysis into the Stokes and Darcy parts, and, on the other hand, these conditions cannot be neglected since they also have to be incorporated into the resulting a posteriori error estimate.

The remainder of the paper is organized as follows: in Section 2, we recall from [1] the Stokes-Darcy coupled problem and its continuous and discrete mixed variational formulations. The kernel of the present work is given by Section 3, where we develop the a posteriori error analysis. We employ auxiliary problem, suitable continuous inf-sup conditions, and local approximation properties for to derive a reliable residual-based a posteriori error estimator (Theorem 3.1).

Next, we apply inverse inequalities, triangular inequality, CauchySchwartz inequality, and the localization technique based on simplexbubble and face-bubble functions to show the efficiency of the error estimator (Theorem 3.2).

In a forthcoming paper, we present the results of numerical tests with the finite element methods. Throughout the rest of the paper, we utilize the standard terminology for Sobolev spaces. In particular, if $S$ is an open set, its closure, or a Lipschitz continuous curve, and $r \in \mathbb{R}$, then $|\cdot|_{r, S}$ and $\|\cdot\|_{r, S}$ stand for the seminorm and norm in the Sobolev spaces $H^{r}(S),\left[H^{r}(S)\right]^{d}$, and $\left[H^{r}(S)\right]^{d \times d}$. Hereafter, given any normed space $U, U^{d}$ and $U^{d \times d}$ denote, respectively, the space of vectors and square matrices of order $d$ with entries in $U$. Also, we employ $\mathbf{0}$ as a generic null vector.

Finally, let $\mathbb{P}_{k}$ be the space of polynomial of total degree not larger than $k$. In order to avoid excessive use of constants, the abbreviations $x \lesssim y$ and $x \sim y$ stand for $x \leq c y$ and $c_{1} x \leq y \leq c_{2} x$, respectively, with positive constants independent of $x, y$ or $\mathcal{T}^{h}$. 


\section{The Stokes-Darcy Coupled Problem}

\subsection{The model problem}

The model we consider consists of Stokes flow in the fluid region $\Omega_{1} \subset \mathbb{R}^{d}$ and Darcy's law in the porous medium domain $\Omega_{2} \subset \mathbb{R}^{d}$ (where $d=2,3$ ). These are separated by an interface $\Gamma_{I}$. Here $\Omega_{j} \subset \mathbb{R}^{d}$, $(j=1,2)$ are bounded domains with outward unit normal vectors $\mathbf{n}_{j}, j=1,2$. Let $\Gamma_{j}:=\partial \Omega_{j} \backslash \Gamma_{I}$. Each interface and boundary is assumed to be polygonal.

The fluid velocities and pressures in $\Omega_{1}$ and $\Omega_{2}$ are denoted by:

$$
\begin{gathered}
\mathbf{u}_{j}: \Omega_{j} \rightarrow \mathbb{R}^{d} \text {, fluid velocity in } \Omega_{j}, \\
p_{j}: \Omega_{j} \rightarrow \mathbb{R} \text {, fluid pressure in } \Omega_{j} .
\end{gathered}
$$

It is important to keep in mind that the velocities and pressures play different mathematical (and physical) roles in the fluid region and in the porous medium.

Recall that the deformation rate tensor $\mathbf{D}$ and stress tensor $\Phi$ associated with $\left(\mathbf{u}_{1}, p_{1}\right)$ are defined by:

$$
\begin{gathered}
\mathbf{D}\left(\mathbf{u}_{1}\right):=\frac{1}{2}\left(\frac{\partial \mathbf{u}_{1 i}}{\partial x_{j}}+\frac{\partial \mathbf{u}_{1 j}}{\partial x_{i}}\right)_{i, j \in\{1, \ldots, d\}} \text { in } \Omega_{1}, \\
\Phi\left(\mathbf{u}_{1}, p_{1}\right):=-p_{1} \mathbf{I}+2 \mu \mathbf{D}\left(\mathbf{u}_{1}\right) \text { in } \Omega_{1},
\end{gathered}
$$

where $\mu$ is the viscosity of fluid. Assuming Stokes flow, $\left(\mathbf{u}_{1}, p_{1}\right)$ satisfies on $\Omega_{1}$ :

$$
\left\{\begin{array}{ccccc}
-\nabla \cdot \Phi\left(\mathbf{u}_{1}, p_{1}\right)=\mathbf{f}_{1} & \text { in } & \Omega_{1} & & \text { (conservation of momentum), } \\
\nabla \cdot \mathbf{u}_{1} & =\mathbf{0} & \text { in } & \Omega_{1} & \text { (conservation of mass), } \\
\mathbf{u}_{1}=\mathbf{0} & \text { on } & \Gamma_{1} & \text { (no slip) },
\end{array}\right.
$$

where $\mathbf{f}_{1}$ is a data which belongs to the space $\left[L^{2}\left(\Omega_{1}\right)\right]^{d}$. 
Assuming Darcy's law and no flow through $\Gamma_{2},\left(\mathbf{u}_{2}, p_{2}\right)$ satisfies on $\Omega_{2}$ :

$\left\{\begin{array}{cccccc}\mathbf{u}_{2} & = & -\mathbf{K} \nabla p_{2} & \text { in } & \Omega_{2} & \text { (Darcy's law) }, \\ \nabla \cdot \mathbf{u}_{2} & = & f_{2} & \text { in } & \Omega_{2} & \text { (conservation of mass), } \\ \mathbf{u}_{2} \cdot \mathbf{n}_{2} & = & 0 & \text { on } & \Gamma_{2} & \text { (no flow) },\end{array}\right.$

where $\mathbf{K}$ is a symmetric and uniformly positive definite tensor representing the rock permeability divided by the fluid viscosity. The source $f_{2}$ is assumed to satisfy the solvability condition:

$$
\int_{\Omega_{2}} f_{2} d x=0
$$

which makes physical sense due to the no-flow boundary condition on $\partial \Omega_{2}$ and to (6) below. The mixed formulation (4) is the most natural one for computations in the porous medium region since it leads to direct approximation of the velocity.

\section{Interface conditions}

The problem (3)-(4) must be coupled across $\Gamma_{I}$ by the correct interface conditions. Mass conservation across $\Gamma_{I}$ is expressed by:

$$
\mathbf{u}_{1} \cdot \mathbf{n}_{1}+\mathbf{u}_{2} \cdot \mathbf{n}_{2}=0 \text { on } \Gamma_{I}
$$

The second interface condition is balance of normal forces across $\Gamma_{I}$ :

$$
p_{1}-2 \mu \mathbf{n}_{1} \cdot \mathbf{D}\left(\mathbf{u}_{1}\right) \cdot \mathbf{n}_{1}=p_{2} \text { on } \Gamma_{I} .
$$

The back interface condition is now know as the Beavers-Joseph-Saffman law whose that:

$$
2 \mu \mathbf{n}_{1} \cdot \mathbf{D}\left(\mathbf{u}_{1}\right) \cdot \tau_{j}=-\frac{\mu}{k_{j}} \mathbf{u}_{1} \cdot \tau_{j} \text { on } \Gamma_{I}, \text { where } j=1, \ldots, d-1
$$


The $\tau_{j}$ are the tangentials vectors on $\Gamma_{I}, \kappa_{j}:=\tau_{h} \cdot \mu \mathbf{K} \cdot \tau_{j}>0$ is the friction constant, and the Beavers-Joseph-Saffman law that the slip velocity along $\Gamma_{I}$ is proportional to the shear stress along $\Gamma_{I}$ (assuming also, based on experimental evidences, that $\mathbf{u}_{2} \cdot \tau_{j}$ is negligible).

Remark 2.1. We remark that on $\Gamma_{I}$ :

$$
\begin{gathered}
\boldsymbol{n}_{1} \cdot \Phi\left(\mathbf{u}_{1}, p_{1}\right) \cdot \boldsymbol{n}_{1}=-p_{1}+2 \mu \boldsymbol{n}_{1} \cdot \mathbf{D}\left(\mathbf{u}_{1}\right) \cdot \boldsymbol{n}_{1}, \text { and } \\
\tau_{j} \cdot \Phi\left(\mathbf{u}_{1}, p_{1}\right) \cdot \boldsymbol{n}_{1}=-\frac{\mu}{\kappa_{j}} \mathbf{u}_{1} \cdot \tau_{j} . \\
\Gamma_{1}
\end{gathered}
$$

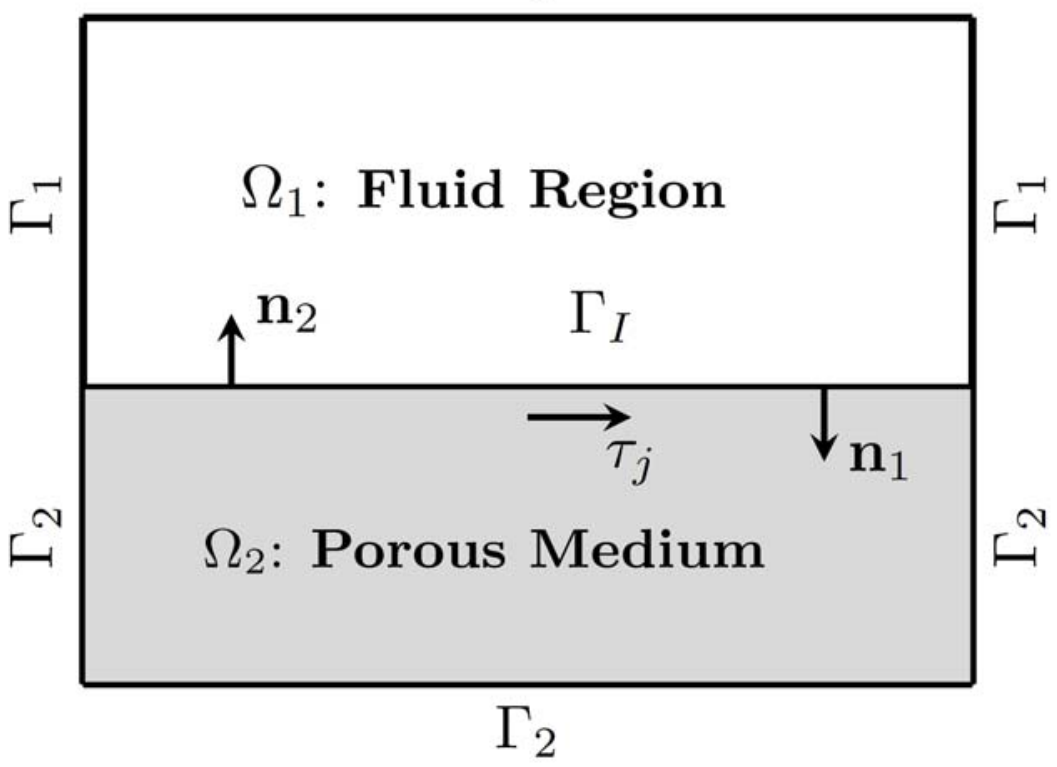

Figure 1. A sketch of the geometry of the problem (case: $\partial \Omega_{d} \neq \Gamma_{I}$ ). 


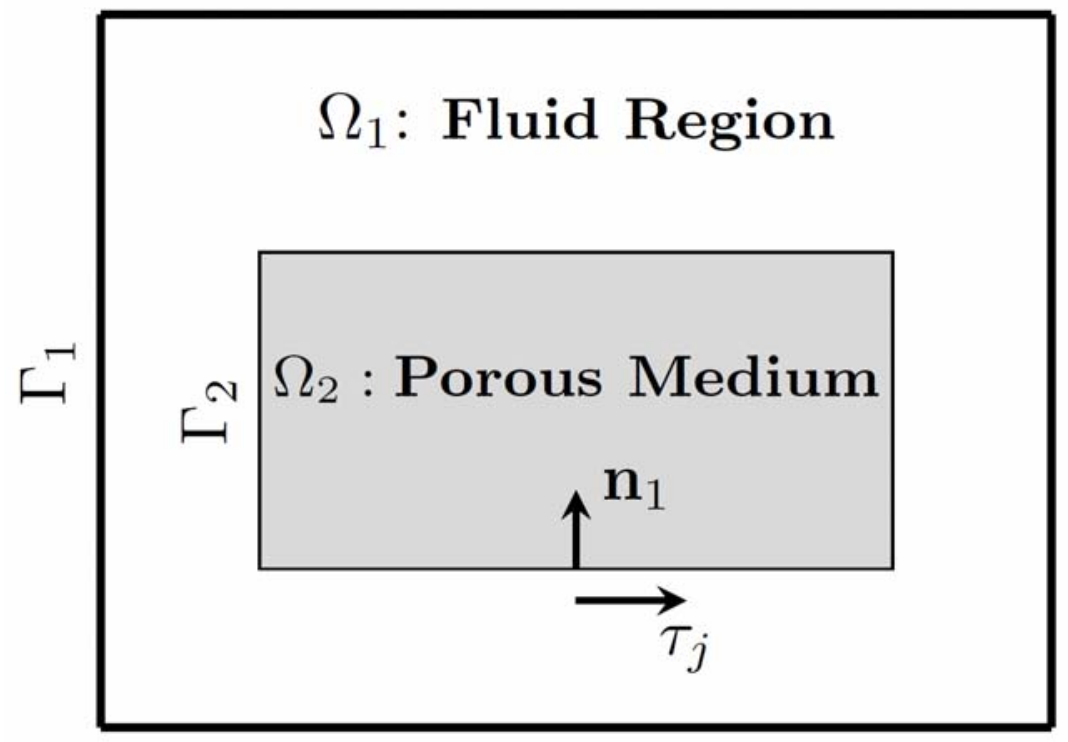

Figure 2. A sketch of the geometry of the problem (case: $\partial \Omega_{d}=\Gamma_{I}$ ).

\subsection{Weak formulation of the coupled problem}

In order to introduce the weak formulation of coupled problem, we define the spaces

$$
\begin{gathered}
\mathbf{H}:=\mathbf{H}_{1} \times \mathbf{H}_{2}, \\
M:=L_{0}^{2}\left(\Omega_{1}\right) \times L_{0}^{2}\left(\Omega_{2}\right), \\
\Lambda:=H^{1 / 2}\left(\Gamma_{I}\right),
\end{gathered}
$$

where

$$
\begin{gathered}
\mathbf{H}_{1}:=\left\{\mathbf{v}_{1} \in\left[H^{1}\left(\Omega_{1}\right)\right]^{d}: \mathbf{v}_{1}=0 \text { on } \Gamma_{1}\right\} \\
\mathbf{H}_{2}:=\left\{\mathbf{v}_{2} \in H\left(\operatorname{div}, \Omega_{2}\right): \mathbf{v}_{2} \cdot \mathbf{n}_{2}=0 \text { on } \Gamma_{2}\right\} .
\end{gathered}
$$

The space $\mathbf{H}$ is equipped the product norm: $\|\mathbf{v}\|_{\mathbf{H}}:=\left\|\mathbf{v}_{1}\right\|_{1, \Omega_{1}}+$ $\left\|\mathbf{v}_{2}\right\|_{H\left(\operatorname{div}, \Omega_{2}\right)}$, for all $\mathbf{v}:=\left(\mathbf{v}_{1}, \mathbf{v}_{2}\right) \in \mathbf{H}$. 
For a connected open subset of the boundary $\Gamma \subset \partial \Omega_{1} \cup \partial \Omega_{2}$, we write $\langle.,\rangle_{\Gamma}$ for the $L^{2}(\Gamma)$ inner product (or duality pairing), that is, for scalar valued functions $\lambda, \eta \in L^{2}(\Gamma)$, one defines

$$
\langle\lambda, \eta\rangle_{\Gamma}:=\int_{\Gamma} \lambda(s) \eta(s) d s
$$

Also, we denote the global unknowns $\mathbf{u}:=\left(\mathbf{u}_{1}, \mathbf{u}_{2}\right), p:=\left(p_{1}, p_{2}\right)$ and introduce the Lagrange multiplier $\lambda:=p_{2}$ on $\Gamma_{I}$. Hence, we proceeding in the usual way (see [1], for example), we find that the mixed variational formulation of coupled problem reads as follows: Find $(\mathbf{u}, p, \lambda) \in \mathbf{H} \times M \times \Lambda$ such that

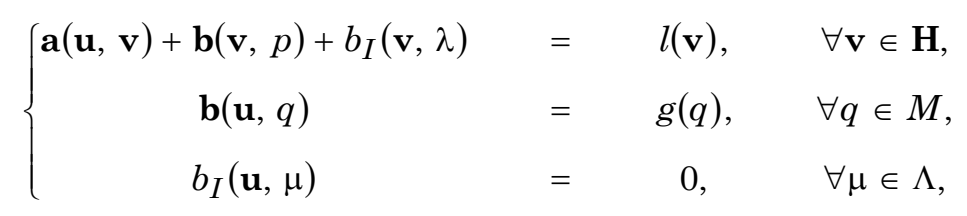

where

$$
\begin{gathered}
b_{I}(\mathbf{v}, \lambda):=<\mathbf{v}_{1} \cdot \mathbf{n}_{1}+\mathbf{v}_{2} \cdot \mathbf{n}_{2}, \lambda>_{\Gamma_{I}}: \mathbf{H} \times \Lambda \rightarrow \mathbb{R} \\
\mathbf{a}(\mathbf{u}, \mathbf{v}):=\sum_{i=1}^{2} a_{i}\left(\mathbf{u}_{i}, \mathbf{v}_{i}\right): \mathbf{H} \times \mathbf{H} \rightarrow \mathbb{R} \\
\mathbf{b}(\mathbf{v}, q):=\sum_{i=1}^{2} b_{i}\left(\mathbf{v}_{i}, q_{i}\right): \mathbf{H} \times M \rightarrow \mathbb{R} \\
l(\mathbf{v}):=\left(\mathbf{f}_{1}, \mathbf{v}_{1}\right)_{\Omega_{1}}, \quad g(q):=-\left(f_{2}, q_{2}\right)_{\Omega_{2}},
\end{gathered}
$$

with

$$
\begin{aligned}
& a_{1}\left(\mathbf{u}_{1}, \mathbf{v}_{1}\right):=2 \mu \int_{\Omega_{1}} \mathbf{D}\left(\mathbf{u}_{1}\right): \mathbf{D}\left(\mathbf{v}_{1}\right)+\sum_{j=1}^{d-1} \frac{\mu \alpha_{1}}{\sqrt{\kappa_{j}}} \int_{\Gamma_{I}}\left(\mathbf{u}_{1}, \tau_{j}\right)\left(\mathbf{v}_{1}, \tau_{j}\right), \\
& a_{2}\left(\mathbf{u}_{2}, \mathbf{v}_{2}\right):=\int_{\Omega_{2}} \mathbf{K}^{-1} \mathbf{u}_{2} \cdot \mathbf{v}_{2}, \\
& b_{1}\left(\mathbf{v}_{1}, q_{1}\right):=-\int_{\Omega_{1}} q_{1} \nabla \cdot \mathbf{v}_{1}, \quad b_{2}\left(\mathbf{v}_{2}, q_{2}\right):=-\int_{\Omega_{2}} q_{2} \nabla \cdot \mathbf{v}_{2} .
\end{aligned}
$$


Here, we use the standard notation for the contraction of two matrices $A$ and $B$, i.e.,

$$
A: B:=\sum_{i, j=1}^{d} A_{i j} B_{i j}
$$

The reason for keeping $H^{1 / 2}\left(\Gamma_{I}\right)$ as the right space for the Lagrange multiplier $\lambda$ which differs from choice of $H_{00}^{1 / 2}\left(\Gamma_{I}\right):=\left[L^{2}\left(\Gamma_{I}\right), H_{0}^{1}\left(\Gamma_{I}\right)\right]_{1 / 2}$ adopted in [1], is that $\lambda$ represents the trace of the porous pressure on $\Gamma_{I}$, and hence there is no physical reason to assume that $\lambda$ vanishes in $\Gamma_{2}$ belong to $H^{1 / 2}\left(\partial \Omega_{2}\right)$. The present choice of $H^{1 / 2}\left(\Gamma_{I}\right)$ is also justified in Subsection 4.1 of [22]. We now recall that, given $\mathbf{v}_{2} \in \mathbf{H}_{2}$, the boundary condition $\mathbf{v}_{2} \cdot \mathbf{n}_{2}=0$ on $\Gamma_{I}$ means:

$$
\left\langle\mathbf{v}_{2} \cdot \mathbf{n}_{2}, E_{00}(\xi)\right\rangle_{\partial \Omega_{2}}=0, \quad \forall \xi \in H_{00}^{1 / 2}\left(\Gamma_{I}\right),
$$

where $E_{00}(\xi)$ denotes the extension by zero in $\Gamma_{I}$ of each $\xi \in H^{1 / 2}\left(\Gamma_{2}\right)$, and $\langle., .\rangle_{\partial \Omega_{2}}$ stands for the duality pairing of $H^{-1 / 2}\left(\partial \Omega_{2}\right)$ and $H^{1 / 2}\left(\partial \Omega_{2}\right)$ with respect to the $L^{2}\left(\partial \Omega_{2}\right)$-inner product.

As a consequence, it is not difficult to show (see ([22], Section 2)) that the restriction of $\mathbf{v}_{2} \cdot \mathbf{n}_{2}$ to $\Gamma_{I}$ can be identified with an element of $H^{-1 / 2}\left(\Gamma_{I}\right)$ :

$$
\left\langle\mathbf{v}_{2} \cdot \mathbf{n}_{2}, \xi\right\rangle_{\Gamma_{I}}:=\left\langle\mathbf{v}_{2} \cdot \mathbf{n}_{2}, E(\xi)\right\rangle_{\partial \Omega_{2}}, \quad \forall \xi \in H^{1 / 2}\left(\Gamma_{I}\right),
$$

where $E: H^{1 / 2}\left(\Gamma_{I}\right) \rightarrow H^{1 / 2}\left(\partial \Omega_{2}\right)$ is the bounded linear operator defined by $E(\xi):=\gamma(z)$ for each $z \in H^{1 / 2}\left(\Gamma_{I}\right), \gamma: H^{1}\left(\Omega_{2}\right) \rightarrow H^{1 / 2}\left(\partial \Omega_{2}\right)$ is the usual trace operator, and $z \in H^{1}\left(\Omega_{2}\right)$ is the unique solution of

$$
\Delta z=0 \text { in } \Omega_{2} ; z=\xi \text { on } \Gamma_{I} \text { and } \nabla z \cdot \mathbf{n}_{2}=0 \text { on } \Gamma_{2} .
$$


Moreover, thanks to (16) and (17), we may also write $\left\langle\mathbf{v}_{2} \cdot \mathbf{n}_{2}, \xi\right\rangle_{\Gamma_{I}}:=\left\langle\mathbf{v}_{2} \cdot\right.$ $\left.\mathbf{n}_{2}, \bar{\xi}\right\rangle_{\partial \Omega_{2}}$, with $\bar{\xi} \in H^{1 / 2}\left(\partial \Omega_{2}\right)$ such that $\bar{\xi}=\xi$ on $\Gamma_{I}$.

In fact, one can prove the following result ([1], Theorem 3.1 and Lemma 3.4).

Theorem 2.1. There exists a unique solution $(\mathbf{u}, p, \lambda)$ to the problem (15).

\subsection{Finite element discretization}

This under section considers the finite element discretization of the coupled problem. We let $\left(\mathcal{T}_{j}^{h}\right)_{h>0}(j=1,2)$ be members of shape-regular families of triangulations, that is, satisfying the minimum angle condition, of $\bar{\Omega}_{1}$ and $\bar{\Omega}_{2}$, respectively, by simplex $T$ of diameter $h_{T}$ (that is $T=$ triangle if $d=2$ and $T=$ tetrahedral if $d=3$ ). Next, we assume that the vertices of $\mathcal{T}_{1}^{h}$ and $\mathcal{T}_{2}^{h}$ coincide on the interface $\Gamma_{I}$. We define $\left(\mathcal{T}^{h}\right)_{h>0}$ a family regular of triangulation on $\bar{\Omega}:=\bar{\Omega}_{1} \cup \Gamma_{I} \cup \bar{\Omega}_{2}$, by $\mathcal{T}^{h}:=\mathcal{T}_{1}^{h} \cup \mathcal{T}_{2}^{h}, \quad$ where $\quad h:=\max \left\{h_{i} ; i=1,2\right\} \quad$ which $\quad h_{i}:=\max$ $\left\{h_{T}, T \in \mathcal{T}_{i}^{h}\right\}$ for each $i \in\{1,2\}$. We use the notation

$$
\begin{gathered}
\varepsilon_{h}(T):=\text { the set of all faces of the elements } K, \\
\varepsilon_{h}\left(\Gamma_{I}\right):=\text { the set of all element faces } E \text { with } E \subset \Gamma_{I} .
\end{gathered}
$$

We now consider $\mathbf{H}_{1}^{h}, \mathbf{H}_{2}^{h}, M_{1}^{h}, M_{2}^{h}$ and $\Lambda^{h}$ be finite dimensional subspaces of $\mathbf{H}_{1}, \mathbf{H}_{2}, L_{0}^{2}\left(\Omega_{1}\right), L_{0}^{2}\left(\Omega_{2}\right)$, and $\Lambda$, respectively. Then, we denote the products spaces as follow $\mathbf{H}^{h}:=\mathbf{H}_{1}^{h} \times \mathbf{H}_{2}^{h}$ and $M^{h}=M_{1}^{h} \times M_{2}^{h}$. 
In this way, the Galerkin schemes of (15) is given by: Find $\left(\mathbf{u}^{h}, p^{h}\right.$, $\left.\lambda^{h}\right) \in \mathbf{H}^{h} \times M^{h} \times \Lambda^{h}$ such that

$$
\left\{\begin{aligned}
\mathbf{a}\left(\mathbf{u}^{h}, \mathbf{v}^{h}\right)+\mathbf{b}\left(\mathbf{v}^{h}, p^{h}\right)+b_{I}\left(\mathbf{v}^{h}, \lambda^{h}\right) & = & l\left(\mathbf{v}^{h}\right), & & \forall \mathbf{v}^{h} \in \mathbf{H}^{h}, \\
\mathbf{b}\left(\mathbf{u}^{h}, q^{h}\right) & = & g\left(q^{h}\right), & & \forall q^{h} \in M^{h}, \\
b_{I}\left(\mathbf{u}^{h}, \xi^{h}\right) & = & 0, & & \forall \xi^{h} \in \Lambda^{h} .
\end{aligned}\right.
$$

Throughout the rest of the subsection, we assume the following hypotheses on the subspaces:

(G.1): For the discretization of the fluid's variables we choose finite element spaces $\mathbf{H}_{1}^{h}, M_{1}^{h}$ which are assumed to be div-stable (also called LBB-stable) i.e., there exists $\beta_{1}>0$ independent of the $h$, such that for each $q_{1}^{h} \in M_{1}^{h}$ there holds

$$
\sup _{\mathbf{v}_{1}^{h} \in \mathbf{H}_{1}^{h}} \frac{b_{1}\left(\mathbf{v}_{1}^{h}, q_{1}^{h}\right)}{\left\|\mathbf{v}_{1}^{h}\right\|_{1, \Omega_{1}}} \geqslant \beta_{1}\left\|q_{1}^{h}\right\|_{0, \Omega_{1}},
$$

and to satisfy a discrete Korn inequality: there exists $\alpha_{1}>0$ independent of the $h$, such that,

$$
\int_{\Omega_{1}} \mathbf{D}\left(\mathbf{v}_{1}^{h}\right): \mathbf{D}\left(\mathbf{v}_{1}^{h}\right) \geqslant \alpha_{1}\left|\mathbf{v}_{1}^{h}\right|_{1, \Omega_{1}} .
$$

In addition, the space of constant functions on $\Omega_{1}$ is contained in $M_{1}^{h}$.

(G.2): For the discretization of the porous medium problem in $\Omega_{2}$, we choose finite element spaces $\mathbf{H}_{2}^{h}, M_{2}^{h}$ which are assumed to be stable, that is, there exists $\beta_{2}>0$, independent of $h$, such that for each $q_{2}^{h} \in M_{2}^{h}$ there holds

$$
\sup _{\mathbf{v}_{2}^{h} \in \mathbf{H}_{2}^{h}} \frac{b_{2}\left(\mathbf{v}_{2}^{h}, q_{2}^{h}\right)}{\left\|\mathbf{v}_{2}^{h}\right\|_{H\left(\operatorname{div}, \Omega_{2}\right)}} \geqslant \beta_{2}\left\|q_{2}^{h}\right\|_{2, \Omega_{2}} .
$$


(G.3): With (G.1) and (G.2), we assume that the space $\Lambda^{h}$ satisfies the inf-sup condition, that is, there exists $\beta_{4}>0$ such that for each $\xi \in \Lambda^{h}$ there holds

$$
\sup _{\mathbf{v} \in \mathbf{H}^{h}} \frac{b_{I}(\mathbf{v}, \xi)}{\|\mathbf{v}\|_{\mathbf{H}}} \geqslant \beta_{4}\|\xi\|_{1 / 2, \Gamma_{I}}
$$

(G.4): Finally, we assume that, there exists an operator $\mathbf{I}^{h}=\left(\mathbf{I}_{1}^{h}, \mathbf{I}_{2}^{h}\right)$, with $\mathbf{I}_{i}^{h}: \mathbf{H}_{i} \rightarrow\left[H^{1}\left(\Omega_{i}\right)\right]^{d}$ such that

$$
\mathbf{I}_{i}^{h}\left(\mathbf{H}_{i}\right) \subset \mathbf{H}_{i}^{h}, \quad i=1,2,
$$

satisfying the local approximation properties:

$$
\begin{gathered}
\left\|\mathbf{v}_{i}-\mathbf{I}_{i}^{h}\left(\mathbf{v}_{i}\right)\right\|_{0, T} \leq C_{1} h_{T}\left\|\mathbf{v}_{i}\right\|_{0, \Delta(T)}, \quad \forall T \in \mathcal{T}_{i}^{h}, i=1,2, \\
\left\|\mathbf{v}_{1}-\mathbf{I}_{1}^{h}\left(\mathbf{v}_{1}\right)\right\|_{0, E} \leq C_{2} h_{E}^{1 / 2}\left\|\mathbf{v}_{1}\right\|_{0, \Delta(E)}, \quad \forall E \in \varepsilon_{h}, \\
\left\|\mathbf{v}_{2} \cdot \mathbf{n}_{2}-\mathbf{I}_{2}^{h}\left(\mathbf{v}_{2}\right) \cdot \mathbf{n}_{2}\right\|_{0, E} \leq C_{3} h_{E}^{1 / 2}\left\|\mathbf{v}_{2}\right\|_{1, \Delta(E)}, \forall E \in \mathcal{T}_{2}^{h}, \quad \forall \mathbf{v}_{2} \in\left[H^{1}\left(\Omega_{2}\right)\right]^{d},
\end{gathered}
$$

where $\Delta(T):=\bigcup\left\{T^{\prime} \in \mathcal{T}_{i}^{h}: T^{\prime} \cap T \neq \emptyset\right\}$ and $\Delta(E):=\bigcup\left\{T^{\prime} \in \mathcal{T}_{i}^{h}: T^{\prime} \cap E \neq \emptyset\right\}$.

Theorem 2.2. Assume that the hypotheses (G.1), (G.2) and (G.3) hold. Then the Galerkin scheme (19) has a unique solution $\left(\mathbf{u}^{h}, p^{h}, \lambda^{h}\right)$ $\in \mathbf{H}^{h} \times M^{h} \times \Lambda^{h}$.

Proof. Cf. [1, 22].

\subsection{Examples of subspaces satisfying the hypotheses}

There is a large variety of stable Stokes elements available in the literature: The Table 1 below provides a list of stable elements covered by our analysis. The first line gives alternative references where some 
equivalences between the error and the residual error estimator have been proved (over kinds of estimators are omitted). In Table 1, the space $\mathbf{S}_{T}$ is Stokes local space, $\mathbf{D}_{T}$ is Darcy local space, and $\mathbf{L}_{T}$ is Lagrange multiplier space. BDM is Brezzi-Douglas-Marini element, BDFM is Brezzi-Douglas-Fortin-Marini element, BDDF is Brezzi Douglas-DuranFortin element and CD is Chen-Douglas element. 
Table 1. Stable isotropic elements covered

\begin{tabular}{|c|c|c|c|c|}
\hline Exaces & Example 1 & Example 2 & Example 3 & Example 4 \\
\hline References & [3] & {$[25,30,31]$} & [33] & [21] \\
\hline $\mathbf{S}_{T}$ & $\begin{array}{c}\text { Mini-Element } \\
(\mathrm{ABF}): \\
{\left[\mathbb{P}^{1}-\text { bulle }\right]^{d} / \mathbb{P}^{1}}\end{array}$ & $\begin{array}{l}\text { Bernardi-Raugel } \\
\qquad(\mathrm{BR}): \\
{\left[\mathbb{P}_{1}\right]^{d} \oplus \text { Enrichi }}\end{array}$ & $\begin{array}{c}\text { Taylor-Hood } \\
\qquad(\mathrm{TH}): \\
{\left[\mathbb{P}_{2}\right]^{d} / \mathbb{P}^{1}}\end{array}$ & $\begin{array}{c}\text { Bernardi-Raugel } \\
\text { (BR): } \\
{\left[\mathbb{P}_{1}\right]^{d} \oplus \text { Enrichi }}\end{array}$ \\
\hline $\mathbf{D}_{T}$ & $\begin{array}{l}\text { Raviart-Thomas } \\
\text { (RT): } \\
\mathbb{P}_{k}(T)^{d} \oplus \mathbb{P}_{k}(T) x \\
k \in \mathbb{N}\end{array}$ & $\begin{array}{l}\text { BDM, BDFM } \\
\text { or } \\
\text { BDDF, CD }\end{array}$ & $\begin{array}{c}\text { Raviart-Thomas } \\
(\mathrm{RT}): \\
\mathbb{P}_{k}(T)^{d} \oplus \mathbb{P}_{k}(T) x \\
k \in \mathbb{N}\end{array}$ & $\begin{array}{c}\text { Raviart-Thomas } \\
\text { (RT): } \\
\mathbb{P}_{k}(T)^{d} \oplus \mathbb{P}_{k}(T) x \\
k \in \mathbb{N}\end{array}$ \\
\hline $\mathbf{L}_{T}$ & $\mathbb{P}_{1}$ - Lagrange & $\mathbb{P}_{1}$ - Lagrange & $\mathbb{P}_{1}$ - Lagrange & $\mathbb{P}_{1}$ - Lagrange \\
\hline
\end{tabular}




\section{Error Estimators}

Here we present our main results, namely reliable and efficient error estimation on isotropic meshes. We will discuss the a posteriori error estimates for finite element approximations of the Darcy-Stokes systems. The upper error bound is derived in Subsection 3.3, whereas the lower error bound is proven in Subsection 3.4. We begin with some notations.

\subsection{Notations}

Given $i \in\{1,2\}$ and $T \in \mathcal{T}_{i}^{h}$, we let $\varepsilon_{h}(T)$ be the set of faces of $T$ and denote by $\varepsilon_{h}$ let the set of all faces of $\mathcal{T}^{h}$. Then we write $\varepsilon_{h}=\varepsilon_{h}\left(\Gamma_{1}\right) \cup \varepsilon_{h}\left(\Omega_{1}\right) \cup \varepsilon_{h}\left(\Gamma_{I}\right) \cup \varepsilon_{h}\left(\Omega_{2}\right) \cup \varepsilon_{h}\left(\Gamma_{2}\right)$, where $\varepsilon_{h}\left(\Gamma_{i}\right):=\left\{E \in \varepsilon_{h}\right.$ $\left.: E \subset \Gamma_{i}\right\}, \varepsilon_{h}\left(\Omega_{i}\right):=\left\{E \in \varepsilon_{h}: E \subset \Omega_{i}\right\}, i=1,2$ and $\varepsilon_{h}\left(\Gamma_{I}\right):=\left\{E \in \varepsilon_{h}\right.$ $\left.: E \subset \Gamma_{I}\right\}$.

Now, let $q \in L^{2}\left(\Omega_{i}\right)$ such that $q_{\mid T} \in C(T)$ for each $T \in \mathcal{T}_{i}^{h}$, and let $E \in \varepsilon_{h}(T) \cap \varepsilon_{h}\left(\Omega_{i}\right)$, we denote by $[q]_{E}$ the jump of $q$ across $E$, that is, $[q]_{E}:=\left(q_{\mid T}-q_{\mid T^{\prime}}\right)_{\mid E}$, where $T^{\prime}$ is the other element of $\mathcal{T}_{i}^{h}$ having $E$ as face. Also, the jump of some (scalar or vector valued) function $\mathbf{v} \in\left[L^{2}\right.$ $\left.\left(\Omega_{i}\right)\right]^{d}, i \in\{1,2\}$ such that $\mathbf{v}_{\mid T} \in[C(T)]^{d}$ defined as $[\mathbf{v}]_{E}:=\left(\mathbf{v}_{\mid T}-\mathbf{v}_{\mid T^{\prime}}\right)_{\mid E}$.

In this next following, we denote by $(\mathbf{u}, p, \lambda)$, with $\mathbf{u}=\left(\mathbf{u}_{1}, \mathbf{u}_{2}\right)$ and $p=\left(p_{1}, p_{2}\right)$ the unique solvability of continuous problem (15). Also, we denote by $\left(\mathbf{u}^{h}, p^{h}, \lambda^{h}\right)$, with $\mathbf{u}^{h}=\left(\mathbf{u}_{1}^{h}, \mathbf{u}_{2}^{h}\right)$ and $p^{h}=\left(p_{1}^{h}, p_{2}^{h}\right)$ the unique solvability of approach problem (19).

\subsection{Residual error estimators}

The general philosophy of residual error estimators is to estimate an appropriate norm of the correct residual by terms that can be evaluated easier, and that involve the data at hand. 
Definition 3.1 (Residual error estimator). For each $T \in \mathcal{T}^{h}$, we define the based-residual errors indicator $\Theta_{i, T}, i \in\{1,2\}$ by:

$$
\begin{aligned}
\Theta_{1, T}:= & h_{T}\left\|\boldsymbol{f}_{1}^{h}+\nabla \cdot \Phi_{1, h}\right\|_{0, T}+\left\|\nabla \cdot \boldsymbol{u}_{1}^{h}\right\|_{0, T} \\
& +\sum_{E \in \varepsilon_{h}(T) \cap \varepsilon_{h}\left(\Gamma_{I}\right)} h_{E}^{1 / 2}\left\|\left(\boldsymbol{n}_{1} \cdot \Phi_{1, h} \cdot \boldsymbol{n}_{1}+\lambda^{h}\right) \boldsymbol{n}_{1}\right\|_{0, E} \\
& +\sum_{E \in \varepsilon_{h}(T) \cap_{\varepsilon_{h}}\left(\Gamma_{I}\right)} h_{E}^{1 / 2}\left\|\sum_{j=1}^{d-1}\left(\boldsymbol{n}_{1} \cdot \Phi_{1, h} \cdot \tau_{j}+\frac{\mu}{\kappa_{j}} \boldsymbol{u}_{1}^{h} \cdot \tau_{j}\right) \tau_{j}\right\|_{0, E} \\
& +\sum_{E \in \varepsilon_{h}(T) \cap \varepsilon_{h}\left(\Gamma_{I}\right)}\left\|\boldsymbol{u}_{1}^{h} \cdot \boldsymbol{n}_{1}+\boldsymbol{u}_{2}^{h} \cdot \boldsymbol{n}_{2}\right\|_{0, E} \\
& +\frac{1}{2} \sum_{E \in \varepsilon_{h}(T) \cap_{\varepsilon_{h}}\left(\Omega_{1}\right)} h_{E}^{1 / 2}\left\|\left[\Phi_{1, h} \cdot \boldsymbol{n}_{1}+2 \mu \sum_{j=1}^{d-1}\left(\tau_{j} \cdot \Phi_{1, h} \cdot \boldsymbol{n}_{1}\right) \tau_{j}\right]_{E}\right\|_{0, E}, \forall T \in \mathcal{T}_{1}^{h},
\end{aligned}
$$

where $\Phi_{1, h}$ is defined by

$$
\Phi_{1, h}:=-p_{1}^{h} I+2 \mu \mathbf{D}\left(\boldsymbol{u}_{1}^{h}\right) \text { on } T \in \mathcal{T}_{1}^{h},
$$

and

$$
\begin{aligned}
\Theta_{2, T}:= & h_{T}\left\|\nabla p_{2}^{h}+\mathbf{K}^{-1} \boldsymbol{u}_{2}^{h}\right\|_{0, T}+\left\|f_{2}^{h}-\nabla \cdot \boldsymbol{u}_{2}^{h}\right\|_{0, T} \\
& +\sum_{E \in \varepsilon_{h}(T) \cap_{\varepsilon_{h}}\left(\Gamma_{I}\right)} h_{E}^{1 / 2}\left\|\left(p_{2}^{h}-\lambda^{h}\right) n_{2}\right\|_{0, E} \\
& +\frac{1}{2} \sum_{E \in \varepsilon_{h}\left(\Omega_{2}\right) \cap \varepsilon_{h}(T)} h_{E}^{1 / 2}\left\|\left[p_{2}^{h} \boldsymbol{n}_{2}\right]_{E}\right\|_{0, E} \\
& +\sum_{E \in \varepsilon_{h}\left(\Gamma_{2}\right) \cap \varepsilon_{h}(T)} h_{E}^{1 / 2}\left\|p_{2}^{h} \boldsymbol{n}_{2}\right\|_{0, E}, \forall T \in \mathcal{T}_{2}^{h} .
\end{aligned}
$$


Then, we introduce the global a posteriori error estimator

$$
\Theta:=\left\{\sum_{T \in \mathcal{T}_{1}^{h}} \Theta_{1, T}^{2}+\sum_{T \in \mathcal{T}_{2}^{h}} \Theta_{2, T}^{2}\right\}^{1 / 2} .
$$

Remark 3.1. The residual character of each term on the right-hand sides of (28) and (30) is quite clear (see, the consistance property (38) and the residual equation (39) below).

\subsection{Proof of the upper error bound}

Global upper error bound is given by the theorem:

Theorem 3.1 (Global upper error bound). The following global upper error bound holds:

$$
\left\|\boldsymbol{u}-\boldsymbol{u}^{h}\right\|_{\boldsymbol{H}}+\left\|p-p^{h}\right\|_{M}+\left\|\lambda-\lambda^{h}\right\|_{\boldsymbol{H}^{1 / 2}\left(\Gamma_{I}\right)} \lesssim \Theta+\zeta
$$

with

$$
\zeta:=\left(\sum_{T \in \mathcal{T}_{1}^{h}} h_{T}^{2}\left\|f_{1}-f_{1}^{h}\right\|_{0, T}^{2}+\sum_{T \in \mathcal{T}_{2}^{h}} h_{T}^{2}\left\|f_{2}-f_{2}^{h}\right\|_{0, T}^{2}\right)^{1 / 2}
$$

The constant intervenying in this inequality (i.e., (32)) depends of parameter of regularity of the triangulation.

Proof. For all $U=(\mathbf{u}, p, \lambda) \in \mathbf{Y}$ and $V=(\mathbf{v}, q, \xi) \in \mathbf{Y}$, we define the continuous bilinear form $A: \mathbf{Y} \times \mathbf{Y} \rightarrow \mathbb{R}$ by:

$$
A(U, V):=\mathbf{a}(\mathbf{u}, \mathbf{v})+\mathbf{b}(\mathbf{v}, p)+b_{I}(\mathbf{v}, \lambda)+\mathbf{b}(\mathbf{u}, q)+b_{I}(\mathbf{u}, \xi)
$$

Hence, the problem $(\mathcal{P})$ is equivalent to problem $(\mathcal{Q})$ : Find $U \in \mathbf{Y}$ such that

$$
A(U, V)=G(V), A V \in \mathbf{Y} \text {, where } G(V)=l(\mathbf{v})+g(q) \text {. }
$$


Thus, approach problem $\left(\mathcal{Q}^{h}\right):$ Find $U_{h} \in \mathbf{Y}^{h}$ such that

$$
A\left(U^{h}, V^{h}\right)=G(V),{ }^{h} \quad \forall V^{h} \in \mathbf{Y}^{h} .
$$

Then, (cf. [5], Theorem 2.4, pp. 32): the bilinear form $A$ satisfy the inf-sup condition on $\mathbf{Y} \times \mathbf{Y}$, i.e., there exists a constant $\beta>0$ such that:

$$
\sup _{V \in \mathbf{Y}} \frac{A(U, V)}{\|V\|_{\mathbf{Y}}} \geq \beta\|U\|_{\mathbf{Y}}, \quad \forall U \in \mathbf{Y}
$$

And, we have the consistance property or Galerkin orthogonality relation

$$
A\left(U-U^{h}, V^{h}\right)=0, \quad \forall V^{h} \in \mathbf{Y}^{h} .
$$

Applying the definition of residual, the consistance property (38) and proceeding by integration by parts on each element of meshes, we obtain the residual equations which is given by:

$$
\begin{aligned}
& A\left(U-U_{h}, V\right)=\sum_{T \in \mathcal{T}_{1}^{h}}\left\{\int_{T}\left(\mathbf{f}_{1}-\mathbf{f}_{1}^{h}\right) \cdot\left(\mathbf{v}_{1}-\mathbf{v}_{1}^{h}\right)+\int_{T}\left(\mathbf{f}_{1}^{h}+\nabla \cdot \Phi_{1, h}\right) \cdot\left(\mathbf{v}_{1}-\mathbf{v}_{1}^{h}\right)\right. \\
& +\int_{T} q_{1} \nabla \cdot \mathbf{u}_{1}^{h}-\sum_{E \in \varepsilon_{h}(T) \cap_{\varepsilon_{h}}\left(\Gamma_{I}\right)} \int_{E}\left(\mathbf{n}_{1} \cdot \Phi_{1, h} \cdot \mathbf{n}_{1}+\lambda^{h}\right) \mathbf{n}_{1} \cdot\left(\mathbf{v}_{1}-\mathbf{v}_{1}^{h}\right) \\
& -\sum_{E \in \varepsilon_{h}(T) \cap_{\varepsilon_{h}}\left(\Gamma_{I}\right)} \int_{E} \sum_{j=1}^{d-1}\left(\tau_{j} \cdot \Phi_{1, h} \cdot \mathbf{n}_{1}+\frac{\mu}{\kappa_{j}} \mathbf{u}_{1}^{h} \cdot \tau_{j}\right) \tau_{j} \cdot\left(\mathbf{v}_{1}-\mathbf{v}_{1}^{h}\right) \\
& -\frac{1}{2} \sum_{E \in \varepsilon_{h}\left(\Omega_{1}\right) \cap_{\varepsilon_{h}}(T)} \int_{E}\left[\Phi_{1, h} \mathbf{n}_{1}+2 \mu \sum_{j=1}^{d-1}\left(\tau_{j} \cdot \Phi_{1, h} \cdot \mathbf{n}_{1}\right) \tau_{j}\right]_{E} \cdot\left(\mathbf{v}_{1}-\mathbf{v}_{1}^{h}\right) \\
& \left.\quad-\sum_{E \in \varepsilon_{h}(T) \cap_{\varepsilon_{h}\left(\Gamma_{I}\right)}<\mathbf{u}_{1}^{h} \cdot \mathbf{n}_{1}+\mathbf{u}_{2}^{h} \cdot \mathbf{n}_{2}, \xi>E}\right\} \\
& +\sum_{T \in \mathcal{T}_{2}^{h}} \int_{T}\left(f_{2}-f_{2}^{h}\right) q_{2}-\int_{T}\left(\nabla p_{2}^{h}+\mathbf{K}^{-1} \mathbf{u}_{2}^{h}\right) \cdot\left(\mathbf{v}_{2}-\mathbf{v}_{2}^{h}\right)
\end{aligned}
$$




$$
\begin{gathered}
\text { RESIDUAL-BASED A POSTERIORI ERROR ... } \\
+\int_{T}\left(-f_{2}^{h}+\nabla \cdot \mathbf{u}_{2}^{h}\right) q_{2}+\sum_{E \in \varepsilon_{h}(T) \cap \varepsilon_{h}\left(\Gamma_{I}\right)} \int_{E}\left(p_{2}^{h}-\lambda^{h}\right) \mathbf{n}_{2} \cdot\left(\mathbf{v}_{2}-\mathbf{v}_{2}^{h}\right) \\
\left.+\frac{1}{2} \sum_{E \in \varepsilon_{h}\left(\Omega_{2}\right) \cap \varepsilon_{h}(T)} \int_{E}\left[p_{2}^{h} \mathbf{n}_{2}\right]_{E} \cdot\left(\mathbf{v}_{2}-\mathbf{v}_{2}^{h}\right)+\sum_{E \in \varepsilon_{h}\left(\Gamma_{2}\right) \cap \varepsilon_{h}(T)} \int_{E} p_{2}^{h} \mathbf{n}_{2} \cdot\left(\mathbf{v}_{2}-\mathbf{v}_{2}^{h}\right)\right\}
\end{gathered}
$$

additionally, we have

$$
\begin{aligned}
& \mathbf{b}\left(\mathbf{u}-\mathbf{u}_{h}, q\right)=\int_{\Omega_{1}} q_{1} \nabla \cdot \mathbf{u}_{1}^{h}+\int_{\Omega_{2}}\left(\nabla \cdot \mathbf{u}_{2}^{h}-f_{2}\right) q_{2}, \\
& b_{I}\left(\mathbf{u}-\mathbf{u}_{h}, \xi\right)=-<\mathbf{u}_{1}^{h} \cdot \mathbf{n}_{1}+\mathbf{u}_{2}^{h} \cdot \mathbf{n}_{2}, \xi>_{\Gamma_{I}},
\end{aligned}
$$

$\mathbf{f}_{1}^{h}$ is the approximation of the data $\mathbf{f}_{1}$ in $\left[L^{2}\left(\Omega_{1}\right)\right]^{d}$ space of functions polynomial on each element $T \in \mathcal{T}_{1}^{h}$ and $f_{2}^{h}$ is the approximation of the data $f_{2}$ in $L^{2}\left(\Omega_{2}\right)$ spaces polynomial on each element $T \in \mathcal{T}_{2}^{h}$.

We apply respectively the triangular inequality and CauchySchwartz inequality to the residual equation (39). Next, we use respectively the interpolation operators of the assumption (G.4) and the inf-sup condition (37), replacing $U$ by $U-U^{h}$. We omit the details.

\subsection{Proof of the lower error bound}

In order to derive the upper bounds for the remaining terms defining the a posteriori error indicator $\Theta_{i, T} i \in\{1,2\}$, we proceed similarly as in [19] and [20] (see also [21]), and apply inverse inequalities, and the localization technique based on simplex-bubble and face-bubble functions. To this end, we now recall some notation and introduce further preliminary results. Given $T \in \mathcal{T}^{h}$, and $E \in \varepsilon_{h}(T)$, we let $b_{T}$ and $b_{E}$ be the usual simplex-bubble and face-bubble functions, respectively (see (1.5) and (1.6) in [3]). In particular, $b_{T}$ satisfies $b_{T} \in \mathbb{P}_{3}(T), \operatorname{supp}\left(b_{T}\right) \subseteq T, b_{T}=0$ over $\partial T, \quad$ and $\quad 0 \leq b_{T} \leq 1 \quad$ on $T$. 
Similarly, $b_{E} \in \mathbb{P}_{2}(T), \operatorname{supp}\left(b_{E}\right) \subseteq W_{E}:=\bigcup\left\{T^{\prime}: E \in \varepsilon_{h}\left(T^{\prime}\right)\right\}, b_{E}=0$ on $\partial T \backslash E$ and $0 \leq b_{E} \leq 1$ in $W_{E}$. We also recall from [4] that, given $k \in \mathbb{N}$, there exists an extension operator $L: C(E) \rightarrow C(T)$ that satisfies $L(p) \in \mathbb{P}_{k}(T)$ and $L(p)_{\mid E}=p, \forall p \in \mathbb{P}_{k}(E)$. A corresponding vectorial version of $L$, that is, the componentwise application of $L$, is denoted by $\mathbf{L}$. Additional properties of $b_{T}, b_{E}$ and $L$ are collected in the following lemma [4].

Lemma 3.1. Given $k \in \mathbb{N}^{*}$, there exist positive constants depending only on $k$ and shape-regularity of the triangulations (minimum angle condition), such that for each simplex $T$ and $E \in \varepsilon_{h}(T)$ there hold

$$
\begin{aligned}
\|q\|_{0, T} & \lesssim\left\|q b_{T}^{1 / 2}\right\|_{0, T} \lessgtr\|q\|_{0, T}, \forall q \in \mathbb{P}_{k}(T), \\
|q|_{1, T} & \lesssim h_{T}^{-1}\|q\|_{0, T}, \forall q \in \mathbb{P}_{k}(T), \\
\|p\|_{0, E} & \lesssim\left\|b_{E}^{1 / 2} p\right\|_{0, E} \preccurlyeq\|p\|_{0, E}, \forall p \in \mathbb{P}_{k}(E), \\
\|L(p)\|_{0, T}+h_{E}|L(p)|_{1, T} & \lesssim h_{E}^{1 / 2}\|p\|_{0, E}, \forall p \in \mathbb{P}_{k}(E) .
\end{aligned}
$$

\subsubsection{Lower error bound in $\Omega_{1}$}

The lower error bound in $\Omega_{1}$ is given by the following proposition:

Proposition 3.1 (Local lower error bound in $\Omega_{1}$ ). For each $T \in \mathcal{T}_{1}^{h}$, the following local lower error bound holds:

$$
\begin{aligned}
\Theta_{1, T} \lesssim & \left\{\left\|\boldsymbol{u}_{1}-\boldsymbol{u}_{1}^{h}\right\|_{1, W_{1}^{T}}+\left\|\boldsymbol{u}_{2}-\boldsymbol{u}_{2}^{h}\right\|_{0, W_{1}^{T}}+\left\|p_{1}-p_{1}^{h}\right\|_{0, W_{1}^{T}}\right. \\
& +\sum_{k \in W_{1}^{T}} h_{k}\left(\left\|\lambda-\lambda^{h}\right\|_{0, \Gamma_{1} \cup \partial k}+\left\|\boldsymbol{u}_{1}-\boldsymbol{u}_{1}^{h}\right\|_{1, k}+\left\|p_{1}-p_{1}^{h}\right\|_{0, k}\right. \\
& \left.\left.+\left\|\nabla \cdot\left(\boldsymbol{u}_{2}-\boldsymbol{u}_{2}^{h}\right)\right\|_{0, k}+\left\|\boldsymbol{f}_{1}-\boldsymbol{f}_{1}^{h}\right\|_{0, k}\right)\right\}
\end{aligned}
$$


where $W_{1}^{T}$ is defined as follow:

$$
W_{1}^{T}:=\left\{T^{\prime} \in \mathcal{T}_{1}^{h}: \partial T \cap \partial T^{\prime} \in \varepsilon_{h}\left(\bar{\Omega}_{1}\right)\right\}
$$

Proof. We begin by bounding each term of the residuals separately.

- To estimate $h_{T}\left\|\mathbf{f}_{1}^{h}+\nabla \cdot \Phi_{1, h}\right\|_{0, T}$. For each $T \in \mathcal{T}_{1}^{h}$, we choose in residual equation (39), $V=\left(\mathbf{v}^{T}, 0,0\right)$ and $\mathbf{v}^{h}=(\mathbf{0}, \mathbf{0})$, with $\mathbf{v}^{T}=\left(\mathbf{v}_{1}^{T}, \mathbf{v}_{2}^{T}\right)$ and $\mathbf{v}_{2}^{T}=\mathbf{0}$ on $\Omega_{2}$,

$$
\mathbf{v}_{1}^{T}:=\left\{\begin{array}{ccc}
\left(\mathbf{f}_{1}^{h}+\nabla \cdot \Phi_{1, h}\right) b_{T} & \text { on } & T \in \mathcal{T}_{1}^{h}, \\
\mathbf{0} & \text { on } & \Omega \backslash T .
\end{array}\right.
$$

We have well $V \in \mathbf{Y}$ and the residual equation (39) becomes:

$$
A\left(U-U_{h}, V\right)=\int_{T}\left(\mathbf{f}_{1}-\mathbf{f}_{1}^{h}\right) \cdot \mathbf{v}_{1}^{T}+\int_{T}\left(\mathbf{f}_{1}^{h}+\nabla \cdot \Phi_{1, h}\right) \cdot \mathbf{v}_{1}^{T},
$$

because the bubble-function $b_{T}$ is vanish on $\partial T$.

$$
\int_{T}\left(\mathbf{f}_{1}^{h}+\nabla \cdot \Phi_{1, h}\right) \cdot \mathbf{v}_{1}^{T}=A\left(U-U_{h}, V\right)-\int_{T}\left(\mathbf{f}_{1}-\mathbf{f}_{1}^{h}\right) \cdot \mathbf{v}_{1}^{T}
$$

On the other hand, using the definition of operator $A$, we have

$$
\begin{aligned}
\int_{T}\left(\mathbf{f}_{1}^{h}+\nabla \cdot \Phi_{1, h}\right) \cdot \mathbf{v}_{1}^{T}=2 \mu \int_{T} \mathbf{D}\left(\mathbf{u}_{1}-\mathbf{u}_{1}^{h}\right): & \mathbf{D}\left(\mathbf{v}_{1}^{T}\right)-\int_{T}\left(p_{1}-p_{1}^{h}\right) \nabla \cdot \mathbf{v}_{1}^{T} \\
& -\int_{T}\left(\mathbf{f}_{1}-\mathbf{f}_{1}^{h}\right) \cdot \mathbf{v}_{1}^{T} \cdot
\end{aligned}
$$

Applying respectively the triangular inequality and the Cauchy-Schwartz inequality to (50), we have

$$
\begin{aligned}
\int_{T}\left(\mathbf{f}_{1}^{h}+\nabla \cdot \Phi_{1, h}\right) \cdot \mathbf{v}_{1}^{T} \lesssim & \left(\left\|\mathbf{u}_{1}-\mathbf{u}_{1}^{h}\right\|_{1, T}+\left\|p_{1}-p_{1}^{h}\right\|_{0, T}\right) \\
& \times\left\|\mathbf{v}_{1}^{T}\right\|_{1, T}+\left\|\mathbf{f}_{1}-\mathbf{f}_{1}^{h}\right\|_{0, T} \times\left\|\mathbf{v}_{1}^{T}\right\|_{0, T} .
\end{aligned}
$$


Next, we use the inverse inequality (43), we have

$$
\begin{array}{r}
\left\|\left(\mathbf{f}_{1}^{h}+\nabla \cdot \Phi_{1, h}\right) b_{T}^{1 / 2}\right\|_{0, T}^{2} \lesssim\left\{h_{T}^{-1}\left(\left|\mathbf{u}_{1}-\mathbf{u}_{1}^{h}\right|_{1, T}+\left\|p_{1}-p_{1}^{h}\right\|_{0, T}\right)+\left\|\mathbf{f}_{1}-\mathbf{f}_{1}^{h}\right\|_{0, T}\right\} \\
\times\left\|\mathbf{v}_{1}^{T}\right\|_{0, T} .
\end{array}
$$

Applying the inverse inequality (42), we have the estimation

$$
\begin{aligned}
\left\|\left(\mathbf{f}_{1}^{h}+\nabla \cdot \Phi_{1, h}\right)\right\|_{0, T}^{2} \lesssim\left\{h _ { T } ^ { - 1 } \left(\mid \mathbf{u}_{1}-\right.\right. & \left.\left.\mathbf{u}_{1}^{h}\right|_{1, T}+\left\|p_{1}-p_{1}^{h}\right\|_{0, T}\right) \\
& \left.+\left\|\mathbf{f}_{1}-\mathbf{f}_{1}^{h}\right\|_{0, T}\right\} \times\left\|\mathbf{v}_{1}^{T}\right\|_{0, T} .
\end{aligned}
$$

Finally, the property $0 \leq b_{T} \leq 1$ and the inequality (51), lead to

$$
h_{T}\left\|\mathbf{f}_{1}^{h}+\nabla \cdot \Phi_{1, h}\right\|_{0, T} \lesssim\left(\left|\mathbf{u}_{1}-\mathbf{u}_{1}^{h}\right|_{1, T}+\left\|p_{1}-p_{1}^{h}\right\|_{0, T}+h_{T}\left\|\mathbf{f}_{1}-\mathbf{f}_{1}^{h}\right\|_{0, T}\right) .
$$

- To estimate $\left\|\nabla \cdot \mathbf{u}_{1}^{h}\right\|_{0, T}$.

$$
\left\|\nabla \cdot \mathbf{u}_{1}^{h}\right\|_{0, T} \lesssim\left|\mathbf{u}_{1}-\mathbf{u}_{1}^{h}\right|_{1, T}
$$

- To estimate $\sum_{E \in \varepsilon_{h}(T) \cap \varepsilon_{h}\left(\Gamma_{I}\right)} h_{E}^{1 / 2}\left\|\left(\mathbf{n}_{1} \cdot \Phi_{1, h} \cdot \mathbf{n}_{1}+\lambda^{h}\right) \mathbf{n}_{1}\right\|_{0, E}$. For each $T \in \mathcal{T}^{h}$ and for each $E \in \varepsilon_{h}(T) \cap \varepsilon_{h}\left(\Gamma_{I}\right)$, we choose in residual equation (39), $V=\left(\mathbf{v}^{E}, 0,0\right), \mathbf{v}^{h}=(\mathbf{0}, \mathbf{0})$, with $\mathbf{v}^{E}=\left(\mathbf{v}_{1}^{E}, \mathbf{0}\right)$, where

$$
\mathbf{v}_{1}^{E}:=\left\{\begin{array}{ccc}
\left.\mathbf{L}\left(\mathbf{n}_{1} \cdot \Phi_{1, h} \cdot \mathbf{n}_{1}+\lambda^{h}\right) \mathbf{n}_{1} b_{E}\right) & \text { on } & \mathrm{T}, \\
\mathbf{0} & \text { on } & \Omega \backslash \mathrm{T} .
\end{array}\right.
$$

We noted that the tangential component of $\mathbf{v}_{1}^{E}$ on $E$ are vanish. In this case, the residual equation become:

$$
\begin{array}{r}
A\left(U-U_{h}, V\right)=\int_{T}\left(\mathbf{f}_{1}-\mathbf{f}_{1}^{h}\right) \cdot \mathbf{v}_{1}^{E}+\int_{T}\left(\mathbf{f}_{1}^{h}+\nabla \cdot \Phi_{1, h}\right) \cdot \mathbf{v}_{1}^{E}-\int_{E}\left(\mathbf{n}_{1} \cdot \Phi_{1, h}\right. \\
\left.\cdot \mathbf{n}_{1}+\lambda^{h}\right) \mathbf{n}_{1} \cdot \mathbf{v}_{1}^{E} .
\end{array}
$$


Hence,

$$
\begin{array}{r}
\left.\int_{E}\left(\mathbf{n}_{1} \cdot \Phi_{1, h} \cdot \mathbf{n}_{1}+\lambda^{h}\right) \mathbf{n}_{1} \cdot \mathbf{v}_{1}^{E}=\int_{T}\left(\mathbf{f}_{1}-\mathbf{f}_{1}^{h}\right) \cdot \mathbf{v}_{1}^{E}+\int_{T} \mathbf{f}_{1}^{h}+\nabla \cdot \Phi_{1, h}\right) \cdot \mathbf{v}_{1}^{E} \\
-A\left(U-U_{h}, V\right) .
\end{array}
$$

On the other hand, by definition of the operator $A$, we have

$$
\begin{gathered}
\int_{E}\left(\mathbf{n}_{1} \cdot \Phi_{1, h} \cdot \mathbf{n}_{1}+\lambda^{h}\right) \mathbf{n}_{1} \cdot \mathbf{v}_{1}^{E}=\int_{T}\left(\mathbf{f}_{1}-\mathbf{f}_{1}^{h}\right) \cdot \mathbf{v}_{1}^{E}+\int_{T}\left(\mathbf{f}_{1}^{h}+\nabla \cdot \Phi_{1, h}\right) \cdot \mathbf{v}_{1}^{E} \\
+2 \mu \int_{T} \mathbf{D}\left(\mathbf{u}_{1}-\mathbf{u}_{1}^{h}\right): \mathbf{D}\left(\mathbf{v}_{1}^{E}\right)-\int_{T}\left(p_{1}-p_{1}^{h}\right) \nabla \cdot \mathbf{v}_{1}^{E} \\
-<\mathbf{v}_{1}^{E}, \lambda-\lambda^{h}>_{E}
\end{gathered}
$$

We apply respectively the triangular inequality and the CauchySchwartz inequality to (55):

$$
\begin{gathered}
\int_{E}\left(\mathbf{n}_{1} \cdot \Phi_{1, h} \cdot \mathbf{n}_{1}+\lambda^{h}\right) \mathbf{n}_{1} \cdot \mathbf{v}_{1}^{E} \leq\left(\left\|\mathbf{f}_{1}-\mathbf{f}_{1}^{h}\right\|_{0, T}+\left\|\mathbf{f}_{1}^{h}+\nabla \cdot \Phi_{1, h}\right\|_{0, T}\right) \times\left\|\mathbf{v}_{1}^{E}\right\|_{0, T} \\
\left(2 \mu\left|\mathbf{u}_{1}-\mathbf{u}_{1}^{h}\right|_{1, T}+\left\|p_{1}-p_{1}^{h}\right\|_{0, T}\right) \times\left|\mathbf{v}_{1}^{E}\right|_{1, T}+\left\|\mathbf{v}_{1}^{E} \cdot \mathbf{n}_{1}\right\|_{0, E} \times\left\|\lambda-\lambda^{h}\right\|_{1 / 2, E} .
\end{gathered}
$$

We apply the inverse inequality (45) to $\mathbf{v}_{1}^{E}$, it comes

$$
\begin{aligned}
h_{E}^{1 / 2} \int_{E}\left(\mathbf{n}_{1} \cdot \Phi_{1, h} \cdot \mathbf{n}_{1}\right. & \left.+\lambda^{h}\right) \mathbf{n}_{1} \cdot \mathbf{v}_{1}^{E} \leq C h_{E}\left\|\mathbf{f}_{1}-\mathbf{f}_{1}^{h}\right\|_{0, T}+h_{E}\left\|\mathbf{f}_{1}^{h}+\nabla \cdot \Phi_{1, h}\right\|_{0, T} \\
& +\left\|p_{1}-p_{1}^{h}\right\|_{0, T}+\left|\mathbf{u}_{1}-\mathbf{u}_{1}^{h}\right|_{1, T} \\
& \left.+h_{T}\left\|\lambda-\lambda^{h}\right\|_{1 / 2, T} \times \| \mathbf{n}_{1} \cdot \Phi_{1, h} \cdot \mathbf{n}_{1}+\lambda^{h}\right) \mathbf{n}_{1} \|_{0, E} \cdot \quad \text { (57) }
\end{aligned}
$$

Also, by definition of the operator $\mathbf{L}$, we have

$$
\begin{array}{r}
\left(\mathbf{n}_{1} \cdot \Phi_{1, h} \cdot \mathbf{n}_{1}+\lambda^{h}\right) \mathbf{n}_{1} \cdot \mathbf{v}_{1}^{E}=\left(\mathbf{n}_{1} \cdot \Phi_{1, h} \cdot \mathbf{n}_{1}+\lambda^{h}\right) \mathbf{n}_{1} \times \mathbf{L}\left[\left(\mathbf{n}_{1} \cdot \Phi_{1, h} \cdot \mathbf{n}_{1} \mathbf{n}_{1}\right.\right. \\
\left.\left.+\lambda^{h}\right) \mathbf{n}_{1} b_{E}\right]=\left[\left(\mathbf{n}_{1} \cdot \Phi_{1, h} \cdot \mathbf{n}_{1}+\lambda^{h}\right) \mathbf{n}_{1} b_{E}\right]^{2}
\end{array}
$$


We use the inverse inequality (44), and we have

$$
\begin{aligned}
h_{E}^{1 / 2} \|\left(\mathbf{n}_{1} \cdot \Phi_{1, h} \cdot \mathbf{n}_{1}\right. & \left.+\lambda^{h}\right) \mathbf{n}_{1}\left\|_{0, E} \lesssim h_{E}\right\| \mathbf{f}_{1}-\mathbf{f}_{1}^{h}\left\|_{0, q}+h_{E}\right\| \mathbf{f}_{1}^{h}+\nabla \cdot \Phi_{1, h} \|_{0, T} \\
& +\left\|p_{1}-p_{1}^{h}\right\|_{0, T}+\left|\mathbf{u}_{1}-\mathbf{u}_{1}^{h}\right|_{0, T} \\
& +h_{T}\left\|\lambda-\lambda^{h}\right\|_{1 / 2, T} \cdot\left\|\left(\mathbf{n}_{1} \cdot \Phi_{1, h} \mathbf{n}_{1}+\lambda^{h}\right) \mathbf{n}_{1}\right\|_{0, E} \cdot
\end{aligned}
$$

Using estimation (52) and the fact that $h_{E} \leq h_{T}$ in (58), we lead to

$$
\begin{array}{r}
\sum_{E \in \varepsilon_{h}(T) \varepsilon_{h}\left(\Gamma_{I}\right)} h_{E}^{1 / 2}\left\|\left(\mathbf{n}_{1} \cdot \Phi_{1, h} \cdot \mathbf{n}_{1}+\lambda^{h}\right) \mathbf{n}_{1}\right\|_{0, E} \lesssim\left\{\left|\mathbf{u}_{1}-\mathbf{u}_{1}^{h}\right|_{1, W_{1}^{T}}+\left\|\lambda-\lambda^{h}\right\|_{1 / 2, \partial T \cap \Gamma_{I}}\right. \\
\left.+\left\|p_{1}-p_{1}^{h}\right\|_{0, W_{1}^{T}}+\sum_{k \in W_{1}^{T}} h_{k}\left(\left\|\mathbf{f}_{1}-\mathbf{f}_{1}^{h}\right\|_{0, k}\right)\right\} .(59)
\end{array}
$$

- To estimate $\sum_{E \in \varepsilon_{h}(T) \cap \varepsilon_{h}\left(\Gamma_{I}\right)} h_{E}^{1 / 2} \| \sum_{j=1}^{d-1}\left(\tau_{j} \cdot \Phi_{1, h} \cdot \mathbf{n}_{1}+\frac{\mu}{\kappa_{j}} \mathbf{u}_{1} \cdot \tau_{j}\right)$

$\tau_{j} \|_{0, E}$. For each $T \in \mathcal{T}^{h}$ and for each $E \in \varepsilon_{h}(T) \cap \varepsilon_{h}\left(\Gamma_{I}\right)$, we choose in residual equation (39), $V=\left(\mathbf{v}^{E}, 0,0\right), \mathbf{v}^{h}=\mathbf{0}$ with $\mathbf{v}^{E}=\left(\mathbf{v}_{1}^{E}, \mathbf{0}\right)$ and

$$
\mathbf{v}_{1}^{E}:=\left\{\begin{array}{ccc}
\mathbf{L}\left(\sum_{j=1}^{d-1}\left(\tau_{j} \cdot \Phi_{1, h} \cdot \mathbf{n}_{1}+\frac{\mu}{\kappa_{j}} \mathbf{u}_{1} \cdot \tau_{j}\right) \tau_{j} b_{E}\right) & \text { on } & T, \\
\mathbf{0} & \text { on } & \Omega \backslash T .
\end{array}\right.
$$

We noted that the normal component of $\mathbf{v}_{1}^{E}$ on $E$ are vanish. Hence, the residual equation (39) become:

$$
\begin{aligned}
A\left(U-U_{h}, V\right)=\int_{T}\left(\mathbf{f}_{1}-\mathbf{f}_{1}^{h}\right) \cdot \mathbf{v}_{1}^{E} & +\int_{T}\left(\mathbf{f}_{1}^{h}+\nabla \cdot \Phi_{1, h}\right) \cdot \mathbf{v}_{1}^{E} \\
& -\sum_{j=1}^{d-1} \int_{E}\left(\tau_{j} \cdot \Phi_{1, h} \cdot \mathbf{n}_{1}+\frac{\mu}{\kappa_{j}} \mathbf{u}_{1}^{h} \cdot \tau_{j}\right) \tau_{j} \mathbf{v}_{1}^{E} .
\end{aligned}
$$


Then,

$$
\begin{aligned}
& \sum_{j=1}^{d-1} \int_{E}\left(\tau_{j} \cdot \Phi_{1, h} \cdot \mathbf{n}_{1}+\frac{\mu}{\kappa_{j}} \mathbf{u}_{1}^{h} \cdot \tau_{j}\right) \tau_{j} \cdot \mathbf{v}_{1}^{E}=\int_{T}\left(\mathbf{f}_{1}-\mathbf{f}_{1}^{h}\right) \cdot \mathbf{v}_{1}^{E} \\
& +\int_{T}\left(\mathbf{f}_{1}^{h}+\nabla \cdot \Phi_{1, h}\right) \cdot \mathbf{v}_{1}^{E}-A\left(U-U_{h}, V\right), \\
& A\left(U-U_{h}, V\right)=2 \mu \int_{T} \mathbf{D}\left(\mathbf{u}_{1}-\mathbf{u}_{1}^{h}\right): \mathbf{D}\left(\mathbf{v}_{1}^{E}\right)-\int_{T}\left(p_{1}-p_{1}^{h}\right) \nabla \cdot \mathbf{v}_{1}^{E} \\
& +<\mathbf{v}_{1}^{E} \cdot \mathbf{n}_{1}, \lambda-\lambda^{h}>_{E}+\sum_{j=1}^{d-1} \frac{\mu}{\kappa_{j}} \int_{E}\left(\mathbf{u}_{1}-\mathbf{u}_{1}^{h}\right) \cdot \tau_{j}\left(\mathbf{v}_{1}^{E} \cdot \tau_{j}\right) .
\end{aligned}
$$

Thus,

$$
\begin{aligned}
\sum_{j=1}^{d-1} \int_{E}\left(\tau_{j} \cdot \Phi_{1, h} \cdot \mathbf{n}_{1}+\frac{\mu}{\kappa_{j}} \mathbf{u}_{1}^{h} \cdot \tau_{j}\right) \tau_{j} \cdot \mathbf{v}_{1}^{E}=\int_{T}\left(\mathbf{f}_{1}-\mathbf{f}_{1}^{h}\right) \cdot \mathbf{v}_{1}^{E}+\int_{T}\left(\mathbf{f}_{1}^{h}\right. \\
\left.+\nabla \cdot \Phi_{1, h}\right) \cdot \mathbf{v}_{1}^{E}-2 \mu \int_{T} \mathbf{D}\left(\mathbf{u}_{1}-\mathbf{u}_{1}^{h}\right): \mathbf{D}\left(\mathbf{v}_{1}^{E}\right)+\int_{T}\left(p_{1}-p_{1}^{h}\right) \nabla \cdot \mathbf{v}_{1}^{E} \\
+\sum_{j=1}^{d-1} \frac{\mu}{\kappa_{j}} \int_{E}\left(\mathbf{u}_{1}-\mathbf{u}_{1}^{h}\right) \cdot \tau_{j}\left(\mathbf{v}_{1}^{E} \cdot \tau_{j}\right)+<\mathbf{v}_{1}^{E} \cdot \mathbf{n}_{1}, \lambda-\lambda^{h}>_{E} .
\end{aligned}
$$

We apply respectively the triangular inequality and the CauchySchwartz inequality:

$$
\begin{array}{r}
\sum_{j=1}^{d-1} \int_{E}\left(\tau_{j} \cdot \Phi_{1, h} \cdot \mathbf{n}_{1}+\frac{\mu}{\kappa_{j}} \mathbf{u}_{1}^{h} \cdot \tau_{j}\right) \tau_{j} \cdot \mathbf{v}_{1}^{E} \leq\left(\left\|\mathbf{f}_{1}-\mathbf{f}_{1}^{h}\right\|_{0, T}+\left\|\mathbf{f}_{1}+\nabla \cdot \Phi_{1, h}\right\|_{0, T}\right) \\
\times\left\|\mathbf{v}_{1}^{E}\right\|_{0, E}+\left(2 \mu\left|\mathbf{u}_{1}-\mathbf{u}_{1}^{h}\right|_{1, T}+\left\|p_{1}-p_{1}^{h}\right\|_{0, T} \times\left|\mathbf{v}_{1}^{E}\right|_{1, T}\right. \\
+\sum_{j=1}^{d-1} \frac{\mu}{\kappa_{j}}\left\|\left(\mathbf{u}_{1}-\mathbf{u}_{1}^{h}\right) \cdot \tau_{j}\right\|_{0, E}\left\|\mathbf{v}_{1}^{E} \cdot \tau_{j}\right\|_{0, E}+\left\|\mathbf{v}_{1}^{E} \cdot \mathbf{n}_{1}\right\|_{0, E} \times\left\|\lambda-\lambda^{h}\right\|_{1 / 2, E} .
\end{array}
$$


Let

$$
\begin{aligned}
\sum_{j=1}^{d-1} \int_{E}\left(\tau_{j} \cdot \Phi_{1, h} \cdot \mathbf{n}_{1}\right. & \left.+\frac{\mu}{\kappa_{j}} \mathbf{u}_{1}^{h} \cdot \tau_{j}\right) \tau_{j} \cdot \mathbf{v}_{1}^{E} \leq\left(\left\|\mathbf{f}_{1}-f_{1}^{h}\right\|_{0, T}+\left\|\mathbf{f}_{1}+\nabla \cdot \Phi_{1, h}\right\|_{0, T}\right) \\
& \times\left\|\mathbf{v}_{1}^{E}\right\|_{0, E}+\left(2 \mu\left|\mathbf{u}_{1}-\mathbf{u}_{1}^{h}\right|_{1, T}+\left\|p_{1}-p_{1}^{h}\right\|_{0, T}\right) \times\left|\mathbf{v}_{1}^{E}\right|_{1, T} \\
+ & \left(\sum_{j=1}^{d-1} \frac{\mu}{\kappa_{j}}\left\|\left(\mathbf{u}_{1}-\mathbf{u}_{1}^{h}\right) \cdot \tau_{j}\right\|_{0, E}+\left\|\lambda-\lambda^{h}\right\|_{1 / 2, E}\right) \times\left\|\mathbf{v}_{1}^{E}\right\|_{0, E} .
\end{aligned}
$$

Next, we apply the inverse inequalities (44) and (42) to $\mathbf{v}_{1}^{E}$, it comes:

$$
\begin{array}{r}
\sum_{j=1}^{d-1} \int_{E}\left(\tau_{j} \cdot \Phi_{1, h} \cdot \mathbf{n}_{1}+\frac{\mu}{\kappa_{j}} \mathbf{u}_{1}^{h} \cdot \tau_{j}\right) \tau_{j} \cdot \mathbf{v}_{1}^{E} \lesssim h_{T}\left\|\mathbf{f}_{1}-\mathbf{f}_{1}^{h}\right\|_{0, T}+h_{T} \| \mathbf{f}_{1}+\nabla \\
\cdot \Phi_{1, h}\left\|_{0, T}+\right\| \lambda-\lambda^{h}\left\|_{1 / 2, T}+2 \mu\left|\mathbf{u}_{1}-\mathbf{u}_{1}^{h}\right|_{1, T}+\right\| p_{1}-p_{1}^{h} \|_{0, T} \\
+\sum_{j=1}^{d-1} \frac{\mu}{\kappa_{j}} h_{T}\left\|\mathbf{u}_{1}-\mathbf{u}_{1}^{h}\right\|_{0, T} .
\end{array}
$$

Using the estimation (52), we deduce finally:

$$
\begin{aligned}
& \sum_{E \in \varepsilon_{h}(T) \cap \varepsilon_{h}\left(\Gamma_{I}\right)} h_{E}^{1 / 2}\left\|\sum_{j=1}^{d-1}\left(\tau_{j} \cdot \Phi_{1, h} \mathbf{n}_{1}+\frac{\mu}{\kappa_{j}} \mathbf{u}_{1} \cdot \tau_{j}\right) \tau_{j}\right\|_{0, E}
\end{aligned}
$$

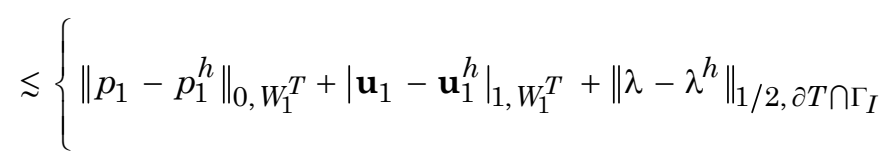

$$
\begin{aligned}
& \left.+\sum_{k \in W_{1}^{T}} h_{k}\left(\left\|\mathbf{f}_{1}-\mathbf{f}_{1}^{h}\right\|_{0, k}+\left\|\mathbf{u}_{1}-\mathbf{u}_{1}^{h}\right\|_{0, k}\right)\right\} .
\end{aligned}
$$


- To estimate $\frac{1}{2} \sum_{E \in \varepsilon_{h}(T) \cap \varepsilon_{h}\left(\Omega_{1}\right)} h_{E}^{1 / 2} \|\left[\Phi_{1, h} \mathbf{n}_{1}+2 \mu \sum_{j=1}^{d-1}\left(\tau_{j} \cdot \Phi_{1, h}\right.\right.$. $\left.\left.\mathbf{n}_{1}\right) \tau_{j}\right]_{E} \|_{0, E}$. For each $T \in \mathcal{T}_{1}^{h}$ and for each $E \in \varepsilon_{h}(T) \cap \varepsilon_{h}\left(\Omega_{1}\right)$, we choose in residual equation (39), $V=\left(\mathbf{v}^{E}, 0,0\right)$ and $\mathbf{v}^{h}=(\mathbf{0}, \mathbf{0})$, with

$$
\begin{aligned}
& \mathbf{v}_{2}^{E}=\mathbf{0} \text { on } \Omega_{2} \text { and } \\
& \mathbf{v}_{1}^{E}:=\left\{\begin{array}{cc}
\mathbf{L}\left(\left[\Phi_{1, h} \cdot \mathbf{n}_{1}+2 \mu \sum_{j=1}^{d-1}\left(\tau_{j} \cdot \Phi_{1, h} \mathbf{n}_{1}\right) \tau_{j}\right]_{E} b_{E}\right) & \text { on } k \in\left\{T, T^{\prime}\right\}, \quad\left(\partial T^{\prime} \cap \partial T=E\right), \\
\mathbf{0} & \text { on } \Omega \backslash T \cup T^{\prime},
\end{array}\right. \\
& A\left(U-U_{h}, V\right)=\int_{T \cup T^{\prime}}\left(\mathbf{f}_{1}-\mathbf{f}_{1}^{h}\right) \cdot \mathbf{v}_{1}^{E}+\int_{T \cup T^{\prime}}\left(\mathbf{f}_{1}^{h}+\nabla \cdot T_{1, h}\right) \cdot \mathbf{v}_{1}^{E} \\
& \quad-\frac{1}{2} \int_{E}\left[\Phi_{1, h} \mathbf{n}_{1}+2 \mu \sum_{j=1}^{d-1}\left(\tau_{j} \cdot \Phi_{1, h} \cdot \mathbf{n}_{1}\right) \tau_{j}\right]_{E} \cdot \mathbf{v}_{1}^{E} .
\end{aligned}
$$

Hence,

$$
\begin{aligned}
\frac{1}{2} \int_{E}\left[\Phi_{1, h} \mathbf{n}_{1}\right. & \left.+2 \mu \sum_{j=1}^{d-1}\left(\tau_{j} \cdot \Phi_{1, h} \cdot \mathbf{n}_{1}\right) \tau_{j}\right]_{E} \cdot \mathbf{v}_{1}^{E}=\int_{T \cup T^{\prime}}\left(\mathbf{f}_{1}-\mathbf{f}_{1}^{h}\right) \cdot \mathbf{v}_{1}^{E} \\
& +\int_{T \cup T^{\prime}}\left(\mathbf{f}_{1}^{h}+\nabla \cdot \Phi_{1, h}\right) \cdot \mathbf{v}_{1}^{E}-A\left(U-U_{h}, V\right) .
\end{aligned}
$$

By definition of operator $A$, we have:

$$
\begin{aligned}
& \quad A\left(U-U_{h}, V\right)=2 \mu \int_{T \cup T^{\prime}} \mathbf{D}\left(\mathbf{u}_{1}-\mathbf{u}_{1}^{h}\right): \mathbf{D}\left(\mathbf{v}_{1}^{E}\right)-\int_{T \cup T^{\prime}}\left(p_{1}-p_{1}^{h}\right) \nabla \cdot \mathbf{v}_{1}^{E}, \\
& \frac{1}{2} \int_{E}\left[\Phi_{1, h} \mathbf{n}_{1}+2 \mu \sum_{j=1}^{d-1}\left(\tau_{j} \cdot \Phi_{1, h} \cdot \mathbf{n}_{1}\right) \tau_{j}\right]_{E} \cdot \mathbf{v}_{1}^{E}=\int_{T \cup T^{\prime}}\left(\mathbf{f}_{1}-\mathbf{f}_{1}^{h}\right) \cdot \mathbf{v}_{1}^{E} \\
& +\int_{T \cup T^{\prime}}\left(\mathbf{f}_{1}^{h}+\nabla \cdot \Phi_{1, h}\right) \cdot \mathbf{v}_{1}^{E}-2 \mu \int_{T \cup T^{\prime}} \mathbf{D}\left(\mathbf{u}_{1}-\mathbf{u}_{1}^{h}\right): \mathbf{D}\left(\mathbf{v}_{1}^{E}\right) \\
& +\int_{T \cup T^{\prime}}\left(p_{1}-p_{1}^{h}\right) \nabla \cdot \mathbf{v}_{1}^{E} .
\end{aligned}
$$


We apply respectively triangular inequality and Cauchy-Schwartz inequality:

$$
\begin{array}{r}
\frac{1}{2} \int_{E}\left[\Phi_{1, h} \mathbf{n}_{1}+2 \mu \sum_{j=1}^{d-1}\left(\tau_{j} \cdot \Phi_{1, h} \mathbf{n}_{1}\right) \tau_{j}\right]_{E} \cdot \mathbf{v}_{1}^{E} \lesssim\left\|\mathbf{f}_{1}-\mathbf{f}_{1}^{h}\right\|_{0, T \cup T^{\prime}} \times\left\|\mathbf{v}_{1}^{E}\right\|_{0, T \cup T^{\prime}} \\
+\left\|\mathbf{f}_{1}^{h}+\nabla \cdot \Phi_{1, h}\right\|_{0, T \cup T^{\prime}} \times\left\|\mathbf{v}_{1}^{E}\right\|_{0, T \cup T^{\prime}} \\
+2 \mu\left|\mathbf{u}_{1}-\mathbf{u}_{1}^{h}\right|_{1, T \cup T^{\prime}} \times\left|\mathbf{v}_{1}^{E}\right|_{1, T \cup T^{\prime}} \\
+\left\|p_{1}-p_{1}^{h}\right\|_{0, T \cup T^{\prime}} \times\left|\mathbf{v}_{1}^{E}\right|_{1, T \cup T^{\prime}}, \\
\frac{1}{2} \int_{E}\left[\Phi_{1, h} \mathbf{n}_{1}+2 \mu \sum_{j=1}^{d-1}\left(\tau_{j} \cdot \Phi_{1, h} \cdot \mathbf{n}_{1}\right) \tau_{j}\right]_{E} \cdot \mathbf{v}_{1}^{E} \lesssim \sum_{k \in\left\{T, T^{\prime}\right\}}\left\{\mid \mathbf{f}_{1}-\mathbf{f}_{1}^{h}\left\|_{0, k}\right\| \mathbf{v}_{1}^{E} \|_{0, k}\right. \\
+\left\|\mathbf{f}_{1}^{h}+\nabla \cdot \Phi_{1, h}\right\|_{0, k} \times\left\|\mathbf{v}_{1}^{E}\right\|_{0, k} \\
\left.+2 \mu\left|\mathbf{u}_{1}-\mathbf{u}_{1}^{h}\right|_{1, k} \times\left|\mathbf{v}_{1}^{E}\right|_{1, k}+\left\|p_{1}-p_{1}^{h}\right\|_{0, k} \times\left|\mathbf{v}_{1}^{E}\right|_{1, k}\right\} .
\end{array}
$$

The inverse inequality (45) gives:

$$
\begin{aligned}
\frac{1}{2} h_{E}^{1 / 2} \int_{E}\left[\Phi_{1, h} \mathbf{n}_{1}\right. & \left.+2 \mu \sum_{j=1}^{d-1}\left(\tau_{j} \cdot \Phi_{1, h} \mathbf{n}_{1}\right) \tau_{j}\right]_{E} \cdot \mathbf{v}_{1}^{E} \lesssim\left(\sum _ { k \in \{ T , T ^ { \prime } \} } \left\{h_{E}\left\|\mathbf{f}_{1}-\mathbf{f}_{1}^{h}\right\|_{0, k}\right.\right. \\
+ & \left.\left.h_{E}\left\|\mathbf{f}_{1}^{h}+\nabla \cdot \Phi_{1, h}\right\|_{0, k}+\left|\mathbf{u}_{1}-\mathbf{u}_{1}^{h}\right|_{0, k}+\left\|p_{1}-p_{1}^{h}\right\|_{0, k}\right\}\right) \\
& \times\left\|\left[\Phi_{1, h} \mathbf{n}_{1}+2 \mu \sum_{j=1}^{d-1}\left(\tau_{j} \cdot \Phi_{1, h} \cdot \mathbf{n}_{1}\right) \tau_{j}\right]_{E}\right\|_{0, E} \cdot
\end{aligned}
$$

Next, we apply the inverse inequality (44) and the definition of the operator L:

$$
\begin{aligned}
\frac{1}{2} h_{E}^{1 / 2} \|\left[\Phi_{1, h} \mathbf{n}_{1}+2 \mu\right. & \left.\sum_{j=1}^{d-1}\left(\tau_{j} \cdot \Phi_{1, h} \cdot \mathbf{n}_{1}\right) \tau_{j}\right]_{E} \|_{0, E} \lesssim\left(\sum _ { k \in \{ T , T ^ { \prime } \} } \left\{h_{k}\left\|\mathbf{f}_{1}-\mathbf{f}_{1}^{h}\right\|_{0, k}\right.\right. \\
& \left.\left.+h_{k}\left\|\mathbf{f}_{1}^{h}+\nabla \cdot \Phi_{1, h}\right\|_{0, k}+\left|\mathbf{u}_{1}-\mathbf{u}_{1}^{h}\right|_{1, k}+\left\|p_{1}-p_{1}^{h}\right\|_{0, k}\right\}\right) .
\end{aligned}
$$


We use the estimation of $h_{k}\left\|\mathbf{f}_{1}^{h}+\nabla \cdot \Phi_{1, h}\right\|_{0, k}$ (cf. (52)), and we have the estimation:

$$
\begin{aligned}
& \frac{1}{2} \sum_{E \in \varepsilon_{h}(T) \cap_{\varepsilon_{h}}\left(\Omega_{1}\right)} h_{E}^{1 / 2}\left\|\left[\Phi_{1, h} \mathbf{n}_{1}+2 \mu \sum_{j=1}^{d-1}\left(\tau_{j} \cdot \Phi_{1, h} \cdot \mathbf{n}_{1}\right) \tau_{j}\right]_{E}\right\|_{o, E} \\
& \lesssim\left(\left|\mathbf{u}_{1}-\mathbf{u}_{1}^{h}\right|_{1, W_{1}^{T}}+\left\|p_{1}-p_{1}^{h}\right\|_{0, W_{1}^{T}}+\sum_{k \in W_{1}^{T}} h_{k}\left\|\mathbf{f}_{1}-\mathbf{f}_{1}^{h}\right\|_{0, k}\right),
\end{aligned}
$$

where $W_{1}^{T}$ is given by (47).

- The following estimation holds for each $T \in \mathcal{T}^{h}$ (cf. [1], Lemma 4.7, pp. 519):

$$
\begin{array}{r}
\sum_{E \in \varepsilon_{h}(T) \cap_{\varepsilon_{h}\left(\Gamma_{1}\right)} h_{E}^{1 / 2}\left\|\mathbf{u}_{1}^{h} \cdot \mathbf{n}_{1}+\mathbf{u}_{2}^{h} \cdot \mathbf{n}_{2}\right\|_{0, E} \lesssim\left\{\left\|\mathbf{u}_{1}-\mathbf{u}_{1}^{h}\right\|_{0, W_{1}^{T}}+\left\|\mathbf{u}_{2}-\mathbf{u}_{2}^{h}\right\|_{0, W_{2}^{T}}\right.} \\
\left.+\sum_{k \in W_{1}^{T}} h_{k}\left|\mathbf{u}_{1}-\mathbf{u}_{1}^{h}\right|_{1, k}+\sum_{k \in W_{2}^{T}}\left\|\nabla \cdot\left(\mathbf{u}_{2}-\mathbf{u}_{2}^{h}\right)\right\|_{0, W_{2}^{T}}\right\} .
\end{array}
$$

Thus, the Proposition 3.1 is proved.

\subsubsection{Local error bound in $\Omega_{2}$}

The local error bound in $\Omega_{2}$ is given by the following proposition: 
Proposition 3.2 (Local lower error bound in $\Omega_{2}$ ). For each $T \in \mathcal{T}_{2}^{h}$, the following local lower error bound holds:

$$
\begin{aligned}
& \Theta_{2, T} \lesssim\left(\left\|\mathbf{u}_{2}-\mathbf{u}_{2}^{h}\right\|_{H\left(\operatorname{div}, W_{2}^{T}\right)}+\left\|p_{2}-p_{2}^{h}\right\|_{0, W_{2}^{T}}\right. \\
& \left.+\sum_{k \in W_{2}^{T}} h_{k}\left(\left\|\mathbf{K}^{-1}\left(\mathbf{u}_{2}-\mathbf{u}_{2}^{h}\right)\right\|_{0, k}+\left\|p_{2}-p_{2}^{h}\right\|_{0, k}+\left\|f_{2}-f_{2}^{h}\right\|_{0, k}+\left\|\lambda-\lambda^{h}\right\|_{0, \Gamma_{I} \cap \partial k}\right)\right) .
\end{aligned}
$$

Proof. We begin also by bounding each term of the residuals separately.

- To estimate $h_{T}\left\|\nabla p_{2}^{h}+\mathbf{K}^{-1} \mathbf{u}_{2}^{h}\right\|_{0, T}$. For each $T \in \mathcal{T}_{2}^{h}$, we choose in the residual equation (39), $V=\left(\mathbf{v}^{T}, 0,0\right)$, with $\mathbf{v}^{T}=\left(\mathbf{0}, \mathbf{v}_{2}^{T}\right)$ and $\mathbf{v}^{h}=(\mathbf{0}, \mathbf{0})$

$$
\mathbf{v}_{2}^{T}:=\left\{\begin{array}{ccc}
\left(\nabla p_{2}^{h}+\mathbf{K}^{-1} \mathbf{u}_{2}^{h}\right) b_{T} & \text { on } & T \in \mathcal{T}_{2}^{h}, \\
\mathbf{0} & \text { on } & \Omega \backslash T .
\end{array}\right.
$$

Hence,

$$
A\left(U-U_{h}, V\right)=-\int_{T}\left(\nabla p_{2}^{h}+\mathbf{K}^{-1} \mathbf{u}_{2}^{h}\right) \cdot \mathbf{v}_{2}^{T} .
$$

Thus

$$
\begin{gathered}
\left\|\left(\nabla p_{2}^{h}+\mathbf{K}^{-1} \mathbf{u}_{2}^{h}\right) b_{T}^{1 / 2}\right\|_{0, T}^{2}=-A\left(U-U_{h}, V\right) \\
\left\|\left(\nabla p_{2}^{h}+\mathbf{K}^{-1} \mathbf{u}_{2}^{h}\right) b_{T}^{1 / 2}\right\|_{0, T}^{2}=-\int_{T} \mathbf{K}^{-1}\left(\mathbf{u}_{2}-\mathbf{u}_{2}^{h}\right) \cdot \mathbf{v}_{2}^{T}+\int_{T}\left(p_{2}-p_{2}^{h}\right) \nabla \cdot \mathbf{v}_{2}^{T} \\
\lesssim\left\|\mathbf{K}^{-1}\left(\mathbf{u}_{2}-\mathbf{u}_{2}^{h}\right)\right\|_{0, T} \times\left\|\mathbf{v}_{2}^{T}\right\|_{0, T}+\left\|p_{2}-p_{2}^{h}\right\|_{0, T} \times\left\|\nabla \cdot \mathbf{v}_{2}^{T}\right\|_{0, T} .
\end{gathered}
$$


We apply the inverse inequality (42) and we get the estimation (65), i.e., the estimate

$$
h_{T}\left\|\nabla p_{2}^{h}+\mathbf{K}^{-1} \mathbf{u}_{2}^{h}\right\|_{0, T} \lesssim h_{T}\left\|\mathbf{K}^{-1}\left(\mathbf{u}_{2}-\mathbf{u}_{2}^{h}\right)\right\|_{0, T}+\left\|p_{2}-p_{2}^{h}\right\|_{0, T} .
$$

- To estimate $\left\|f_{2}^{h}-\nabla \cdot \mathbf{u}_{2}^{h}\right\|_{0, T}$. For each $T \in \mathcal{T}_{2}^{h}$, we choose in the residual equation (39), $V=\left(\mathbf{0}, q^{T}, 0\right)$ and $\mathbf{v}^{h}=(\mathbf{0}, \mathbf{0})$, with $q^{T}=\left(0, q_{2}^{T}\right)$ and

$$
q_{2}^{T}:=\left\{\begin{array}{ccc}
\left(-f_{2}^{h}+\nabla \cdot \mathbf{u}_{2}^{h}\right) b_{T} & \text { on } & T \in \mathcal{T}_{2}^{h}, \\
\mathbf{0} & \text { on } & \Omega \backslash T .
\end{array}\right.
$$

Then, we have

$$
A\left(U-U_{h}, V\right)=\int_{T}\left(f_{2}^{h}-f_{2}\right) q_{2}^{T}+\int_{T}\left(-f_{2}^{h}+\nabla \cdot \mathbf{u}_{2}^{h}\right) q_{2}^{T} .
$$

Let

$$
\begin{aligned}
\int_{T}\left(-f_{2}^{h}+\nabla \cdot \mathbf{u}_{2}^{h}\right) q_{2}^{T} & =A\left(U-U_{h}, V\right)-\int_{T}\left(f_{2}^{h}-f_{2}\right) q_{2}^{T} \\
& =-\int_{T} q_{2}^{T} \nabla \cdot\left(\mathbf{u}_{2}-\mathbf{u}_{2}^{h}\right)-\int_{T}\left(f_{2}-f_{2}^{h}\right) q_{2}^{T} \\
& \leq\left(\left\|\nabla \cdot\left(\mathbf{u}_{2}-\mathbf{u}_{2}^{h}\right)\right\|_{0, T}+\left\|f_{2}-f_{2}^{h}\right\|_{0, T}\right)\left\|q_{2}^{T}\right\|_{0, T} .
\end{aligned}
$$

Hence,

$$
\begin{array}{r}
\left\|\left(-f_{2}^{h}+\nabla \cdot \mathbf{u}_{2}^{h}\right) b_{T}^{1 / 2}\right\|_{0, T}^{2} \leq\left(\left\|\mathbf{u}_{2}-\mathbf{u}_{2}^{h}\right\|_{H(\operatorname{div}, T)}+\left\|f_{2}-f_{2}^{h}\right\|_{0, T} \times \|\left(-f_{2}^{h}\right.\right. \\
\left.\left.+\nabla \cdot \mathbf{u}_{2}^{h}\right) b_{T}\right) \|_{0, T}
\end{array}
$$

The inverse inequality (42) and the property $0 \leq b_{T} \leq 1$ give estimation (67), i.e.,

$$
\left\|f_{2}^{h}-\nabla \cdot \mathbf{u}_{2}^{h}\right\|_{0, T} \lesssim\left(\left\|\mathbf{u}_{2}-\mathbf{u}_{2}^{h}\right\|_{H(\operatorname{div}, T)}+\left\|f_{2}-f_{2}^{h}\right\|_{0, T}\right) .
$$


- To estimate $\sum_{E \in \varepsilon_{h}(T) \cap \varepsilon_{h}\left(\Gamma_{I}\right)} h_{E}^{1 / 2}\left\|\left(p_{2}^{h}-\lambda^{h}\right) \mathbf{n}_{2}\right\|_{0, E}$. For each $T \in \mathcal{T}^{h}$ and for each $E \in \varepsilon_{h}(T) \cap \varepsilon_{h}\left(\Gamma_{I}\right)$, we choose in the residual equation (39), $V=\left(\mathbf{v}^{E}, 0,0\right)$, and $\mathbf{v}^{h}=(\mathbf{0}, \mathbf{0})$, with $\mathbf{v}^{E}=\left(\mathbf{0}, \mathbf{v}_{2}^{E}\right)$,

$$
\mathbf{v}_{2}^{E}:=\left\{\begin{array}{ccc}
\mathbf{L}\left(\left(p_{2}^{h}-\lambda^{h}\right) \mathbf{n}_{2} b_{E}\right) & \text { on } & \mathrm{T}, \\
\mathbf{0} & \text { on } & \Omega \backslash \mathrm{T} .
\end{array}\right.
$$

Then

$$
A\left(U-U_{h}, V\right)=-\int_{T}\left(\nabla p_{2}^{h}+\mathbf{K}^{-1} \mathbf{u}_{2}^{h}\right) \cdot \mathbf{v}_{2}^{E}+\int_{E}\left(p_{2}^{h}-\lambda^{h}\right) \mathbf{n}_{2} \cdot \mathbf{v}_{2}^{E} .
$$

Let

$$
\int_{E}\left(p_{2}-\lambda^{h}\right) \mathbf{n}_{2} \cdot \mathbf{v}_{2}^{E}=A\left(U-U_{h}, V\right)+\int_{T}\left(\nabla p_{2}^{h}+\mathbf{K}^{-1} \mathbf{u}_{2}^{h}\right) \cdot \mathbf{v}_{2}^{E} .
$$

On the other hand,

$$
A\left(U-U_{h}, V\right)=-\int_{T}\left(p_{2}-p_{2}^{h}\right) \nabla \cdot \mathbf{v}_{2}^{E}+<\mathbf{v}_{2}^{E} \cdot \mathbf{n}_{2}, \lambda-\lambda^{h}>_{E} \cdot
$$

Combining (69) and (70), we have

$$
\begin{aligned}
\int_{E}\left(p_{2}-\lambda^{h}\right) \mathbf{n}_{2} \cdot \mathbf{v}_{2}^{E}=-\int_{T}\left(p_{2}-p_{2}^{h}\right) \nabla \cdot \mathbf{v}_{2}^{E}+ & <\mathbf{v}_{2}^{E} \cdot \mathbf{n}_{2}, \lambda-\lambda^{h}>_{E} \\
& +\int_{T}\left(\nabla p_{2}^{h}+\mathbf{K}^{-1} \mathbf{u}_{2}^{h}\right) \cdot \mathbf{v}_{2}^{E} .
\end{aligned}
$$

We use the triangular inequality and the Cauchy-Schwartz inequality:

$$
\begin{aligned}
\int_{E}\left(p_{2}-\lambda^{h}\right) \mathbf{n}_{2} \cdot \mathbf{v}_{2}^{E} & \lesssim\left\|p_{2}-p_{2}^{h}\right\|_{0, T} \cdot\left|\mathbf{v}_{2}^{E}\right|_{1, T}+\left\|\nabla p_{2}^{h}+\mathbf{K}^{-1} \mathbf{u}_{2}^{h}\right\|_{0, T} \\
& \times\left\|\mathbf{v}_{2}^{E}\right\|_{0, T}+\left\|\lambda-\lambda^{h}\right\|_{1 / 2, E} \times\left\|\mathbf{v}_{2}^{E}\right\|_{0, E} .
\end{aligned}
$$


Next, we apply the inverse inequality (45) and $\left(p_{2}^{h}-\lambda^{h}\right) \mathbf{n}_{2}$, it comes

$$
\begin{array}{r}
h_{E}^{1 / 2} \int_{E}\left(p_{2}-\lambda^{h}\right) \mathbf{n}_{2} \cdot \mathbf{v}_{2}^{E} \lesssim\left\{\left\|p_{2}-p_{2}^{h}\right\|_{0, T}+\left\|\left(\lambda-\lambda^{h}\right)\right\|_{0, E}\right. \\
\left.+h_{E}\left\|\nabla p_{2}^{h}+\mathbf{K}^{-1} \mathbf{u}_{2}^{h}\right\|_{0, T}\right\} \times\left\|\left(p_{2}^{h}-\lambda^{h}\right) \mathbf{n}_{2}\right\|_{0, E} .
\end{array}
$$

We combine the inverse inequality (44) with (71), and we get the estimation (72), i.e.,

$$
\begin{array}{r}
\sum_{E \in \varepsilon_{h}(T) \cap \varepsilon_{h}\left(\Gamma_{I}\right)} h_{E}^{1 / 2}\left\|\left(p_{2}^{h}-\lambda^{h}\right) \mathbf{n}_{2}\right\|_{0, E} \lesssim\left\|p_{2}-p_{2}^{h}\right\|_{0, W_{2}^{T}}+\left\|\lambda-\lambda^{h}\right\|_{1 / 2, \partial T \cap \Gamma_{I}} \\
+\sum_{k \in W_{2}^{T}} h_{k}\left(\left\|\mathbf{K}^{-1}\left(\mathbf{u}_{2}-\mathbf{u}_{2}^{h}\right)\right\|_{0, k}+\left\|p_{2}-p_{2}^{h}\right\|_{0, k}\right) .
\end{array}
$$

- To estimate $\frac{1}{2} \sum_{E \in \varepsilon_{h}\left(\Omega_{2}\right) \cap \varepsilon_{h}(T)}\left\|\left[p_{2}^{h} \mathbf{n}_{2}\right]_{E}\right\|_{0, E}$. For each $T \in \mathcal{T}_{2}^{h}$ and for each $E \in \varepsilon_{h}\left(\Omega_{2}\right) \cap \varepsilon_{h}(T)$, we choose in the residual equation (39), $V=\left(\mathbf{v}^{E}, 0,0\right)$ and $\mathbf{v}^{h}=(\mathbf{0}, \mathbf{0})$ with $\mathbf{v}^{E}=\left(\mathbf{0}, \mathbf{v}_{2}^{E}\right)$ and $\mathbf{v}_{2}^{E}:=\left\{\begin{array}{cll}\mathbf{L}\left(\left[p_{2}^{h} \mathbf{n}_{2}\right]_{E} b_{E}\right) & \text { on } & k \in\left\{T, T^{\prime}\right\}, \\ \mathbf{0} & \text { on } & \Omega \backslash T \cup T^{\prime} .\end{array} \quad\left(\right.\right.$ where $\left.\partial T \cap \partial T^{\prime}=E\right)$,

Then, we have

$$
A\left(U-U_{h}, V\right)=-\int_{T \cup T^{\prime}}\left(\nabla p_{2}^{h}+\mathbf{K}^{-1} \mathbf{u}_{2}^{h}\right) \cdot \mathbf{v}_{2}^{E}+\frac{1}{2} \int_{E}\left[p_{2}^{h} \mathbf{n}_{2}\right]_{E} \cdot \mathbf{v}_{2}^{E} .
$$

Hence

$$
\frac{1}{2} \int_{E}\left[p_{2}^{h} \mathbf{n}_{2}\right]_{E} \cdot \mathbf{v}_{2}^{E}=A\left(U-U_{h}, V\right)+\int_{T \cup T^{\prime}}\left(\nabla p_{2}^{h}+\mathbf{K}^{-1} \mathbf{u}_{2}^{h}\right) \cdot \mathbf{v}_{2}^{E}
$$


Using the definition of the operator $A$, we have

$$
\begin{aligned}
\frac{1}{2} \int_{E}\left[p_{2}^{h} \mathbf{n}_{2}\right]_{E} \cdot \mathbf{v}_{2}^{E}=\int_{T \cup T^{\prime}}\left(\nabla p_{2}^{h}+\mathbf{K}^{-1} \mathbf{u}_{2}^{h}\right) \cdot \mathbf{v}_{2}^{E} \\
\quad-\int_{T \cup T^{\prime}} \mathbf{K}^{-1}\left(\mathbf{u}_{2}-\mathbf{u}_{2}^{h}\right) \cdot \mathbf{v}_{2}^{E}+\int_{T \cup T^{\prime}}\left(\nabla p_{2}^{h}+\mathbf{K}^{-1} \mathbf{u}_{2}^{h}\right) \cdot \mathbf{v}_{2}^{E} .
\end{aligned}
$$

We apply respectively to (73), the triangular inequality and the CauchySchwartz inequality:

$$
\begin{aligned}
& \frac{1}{2} \int_{E}\left[p_{2}^{h} \mathbf{n}_{2}\right]_{E} \cdot \mathbf{v}_{2}^{E} \leq\left\|\mathbf{K}^{-1}\left(\mathbf{u}_{2}-\mathbf{u}_{2}^{h}\right)\right\|_{0, T \cup T^{\prime}} \times\left\|\mathbf{v}_{2}^{E}\right\|_{0, T \cup T^{\prime}} \\
& +\left\|p_{2}-p_{2}^{h}\right\|_{0, T \cup T^{\prime}} \times\left|\mathbf{v}_{2}^{E}\right|_{0, T \cup T^{\prime \prime}}+\left\|\nabla p_{2}^{h}+\mathbf{K}^{-1} \mathbf{u}_{2}^{h}\right\|_{0, T \cup T^{\prime}} \times\left\|\mathbf{v}_{2}^{E}\right\|_{0, T \cup T^{\prime}}
\end{aligned}
$$

Let

$$
\begin{aligned}
& \frac{1}{2} \int_{E}\left[p_{2}^{h} \mathbf{n}_{2}\right]_{E} \cdot \mathbf{v}_{2}^{E} \leq \sum_{k \in\left\{T, T^{\prime}\right\}}\left\{\left\|\mathbf{K}^{-1}\left(\mathbf{u}_{2}-\mathbf{u}_{2}^{h}\right)\right\|_{0, k} \times\left\|\mathbf{v}_{2}^{E}\right\|_{0, k}\right. \\
& \left.+\left\|p_{2}-p_{2}^{h}\right\|_{0, k}\left|\mathbf{v}_{2}^{E}\right|_{0, k}+\left\|\nabla p_{2}^{h}+\mathbf{K}^{-1} \mathbf{u}_{2}^{h}\right\|_{0, k} \times\left\|\mathbf{v}_{2}^{E}\right\|_{0, k}\right\}
\end{aligned}
$$

We apply inverse inequality (45):

$$
\begin{aligned}
& \frac{1}{2} h_{E}^{1 / 2} \int_{E}\left[p_{2}^{h} \mathbf{n}_{2}\right]_{E} \cdot \mathbf{v}_{2}^{E} \lesssim \sum_{k \in\left\{T, T^{\prime}\right\}}\left\{h_{k}\left\|\mathbf{K}^{-1}\left(\mathbf{u}_{2}-\mathbf{u}_{2}^{h}\right)\right\|_{0, k}\right. \\
& \left.\quad+\left\|p_{2}-p_{2}^{h}\right\|_{0, k}+h_{k}\left\|\nabla p_{2}^{h}+\mathbf{K}^{-1} \mathbf{u}_{2}^{h}\right\|_{0, k}\right\}\left\|\left[p_{2}^{h} \mathbf{n}_{2}\right]_{E}\right\|_{0, E}
\end{aligned}
$$

On the other hand, $\left[p_{2}^{h} \mathbf{n}_{2}\right]_{E} \cdot \mathbf{v}_{2}^{E}=\left[p_{2}^{h} \mathbf{n}_{2}\right]_{E}^{2} b_{E}$. Hence

$$
\begin{aligned}
\frac{1}{2} h_{E}^{1 / 2}\left\|\left[p_{2}^{h} \mathbf{n}_{2}\right]_{E} b_{E}^{1 / 2}\right\|_{0, E}^{2} \leq & C \sum_{k \in\left\{T, T^{\prime}\right\}}\left\{h_{k}\left\|\mathbf{K}^{-1}\left(\mathbf{u}_{2}-\mathbf{u}_{2}^{h}\right)\right\|_{0, k}+\left\|p_{2}-p_{2}^{h}\right\|_{0, k}\right. \\
& \left.+h_{k}\left\|\nabla p_{2}^{h}+\mathbf{K}^{-1} \mathbf{u}_{2}^{h}\right\|_{0, k}\right\}\left\|\left[p_{2}^{h} \mathbf{n}_{2}\right]_{E}\right\|_{0, E}
\end{aligned}
$$


Next, we apply inverse inequality (44):

$$
\begin{aligned}
\frac{1}{2} h_{E}^{1 / 2}\left\|\left[p_{2}^{h} \mathbf{n}_{2}\right]_{E}\right\|_{0, E} \leq & \sum_{k \in\left\{T, T^{\prime}\right\}}\left\{h_{k}\left\|\mathbf{K}^{-1}\left(\mathbf{u}_{2}-\mathbf{u}_{2}^{h}\right)\right\|_{0, k}+\left\|p_{2}-p_{2}^{h}\right\|_{0, k}\right. \\
& \left.+h_{k}\left\|\nabla p_{2}^{h}+\mathbf{K}^{-1} \mathbf{u}_{2}^{h}\right\|_{0, k}\right\}
\end{aligned}
$$

Finally, we use (65) and adding on $E \in \varepsilon_{h}\left(\Omega_{2}\right) \cap \varepsilon_{h}(T)$, we get the estimation (79), i.e.,

$$
\begin{aligned}
\frac{1}{2} \sum_{E \in \varepsilon_{h}\left(\Omega_{2}\right) \cap \varepsilon_{h}(T)}\left\|\left[p_{2}^{h} \mathbf{n}_{2}\right]_{E}\right\|_{0, E} \lesssim\left\{\left\|p_{2}-p_{2}^{h}\right\|_{0, W_{2}^{T}}\right. \\
\left.\quad+\sum_{k \in W_{2}^{T}} h_{k}\left(\left\|\mathbf{K}^{-1}\left(\mathbf{u}_{2}-\mathbf{u}_{2}^{h}\right)\right\|_{0, k}+\left\|p_{2}-p_{2}^{h}\right\|_{0, k}\right)\right\}
\end{aligned}
$$

where $W_{2}^{T}$ is defined as follow:

$$
W_{2}^{T}:=\left\{T^{\prime} \in \mathcal{T}_{2}^{h}: \partial T \cap \partial T^{\prime} \in \varepsilon_{h}\left(\bar{\Omega}_{2}\right)\right\}
$$

- To estimate $\sum_{E \in \varepsilon_{h}(T) \cap \varepsilon_{h}\left(\Gamma_{2}\right)} h_{E}^{1 / 2}\left\|p_{2} \mathbf{n}_{2}\right\|_{0, E}$. For each $T \in \mathcal{T}_{2}^{h}$ and for each $E \in \varepsilon_{h}(T) \cap \varepsilon_{h}\left(\Gamma_{2}\right)$, we choose in the residual equation (39), $V=\left(\mathbf{v}^{E}, 0,0\right), \mathbf{v}^{h}=(\mathbf{0}, \mathbf{0})$, with $\mathbf{v}^{E}=\left(\mathbf{0}, \mathbf{v}_{2}^{E}\right)$, where

$$
\mathbf{v}_{2}^{E}:=\left\{\begin{array}{ccc}
\mathbf{L}\left(p_{2}^{h} \mathbf{n}_{2} b_{E}\right) & \text { on } & T, \\
\mathbf{0} & \text { on } & \Omega \backslash T .
\end{array}\right.
$$

Then, the residual equation becomes:

$$
A\left(U-U_{h}, V\right)=-\int_{T}\left(\nabla p_{2}^{h}+\mathbf{K}^{-1} \mathbf{u}_{2}^{h}\right) \cdot \mathbf{v}_{2}^{E}+\int_{E} p_{2}^{h} \mathbf{n}_{2} \cdot \mathbf{v}_{2}^{E} .
$$

Hence,

$$
\int_{E} p_{2}^{h} \mathbf{n}_{2} \cdot \mathbf{v}_{2}^{E}=A\left(U-U_{h}, V\right)+\int_{T}\left(\nabla p_{2}^{h}+\mathbf{K}^{-1} \mathbf{u}_{2}^{h}\right) \cdot \mathbf{v}_{2}^{E}
$$


We use the definition of the operator $A$ :

$$
\begin{aligned}
\int_{E} p_{2}^{h} \mathbf{n}_{2} \cdot \mathbf{v}_{2}^{E}=-\int_{T} \mathbf{K}^{-1}\left(\mathbf{u}_{2}-\mathbf{u}_{2}^{h}\right) \cdot v_{2}^{E} & +\int_{T}\left(p_{2}-p_{2}^{h}\right) \nabla \cdot v_{2}^{E} \\
& +\int_{T}\left(\nabla p_{2}^{h}+\mathbf{K}^{-1} \mathbf{u}_{2}^{h}\right) \cdot v_{2}^{E} .
\end{aligned}
$$

We apply respectively, the triangular inequality and the CauchySchwartz inequality:

$$
\begin{aligned}
\left\|p_{2}^{h} \mathbf{n}_{2} b_{E}^{1 / 2}\right\|_{0, E}^{2} \leq & \left\|\mathbf{K}^{-1}\left(\mathbf{u}_{2}-\mathbf{u}_{2}^{h}\right)\right\|_{0, T} \times\left\|v_{2}^{E}\right\|_{0, T} \\
& +\left\|p_{2}-p_{2}^{h}\right\|_{0, T} \times\left\|\nabla \cdot v_{2}^{E}\right\|_{0, T} \\
& +\left\|\nabla p_{2}^{h}+\mathbf{K}^{-1} \mathbf{u}_{2}^{h}\right\|_{0, T} \times\left\|v_{2}^{E}\right\|_{0, T} .
\end{aligned}
$$

Next, we apply inverse inequality (45) to (83):

$$
\begin{aligned}
h_{E}^{1 / 2}\left\|p_{2}^{h} \mathbf{n}_{2} b_{E}^{1 / 2}\right\|_{0, E}^{2} \lesssim & h_{E}^{1 / 2}\left\|\mathbf{K}^{-1}\left(\mathbf{u}_{2}-\mathbf{u}_{2}^{h}\right)\right\|_{0, T} \\
& \times\left\|v_{2}^{E}\right\|_{0, T}+\left\|p_{2}-p_{2}^{h}\right\|_{0, T} \times\left\|p_{2}^{h} \mathbf{n}_{2} b_{E}\right\|_{0, E} \\
& +h_{E}^{1 / 2}\left\|\nabla p_{2}^{h}+\mathbf{K}^{-1} \mathbf{u}_{2}^{h}\right\|_{0, T} \times\left\|v_{2}^{E}\right\|_{0, T} .
\end{aligned}
$$

The inverse inequality (45) gives:

$$
\begin{aligned}
h_{E}^{1 / 2}\left\|p_{2}^{h} \mathbf{n}_{2} b_{E}^{1 / 2}\right\|_{0, E}^{2} \lesssim & \left(h_{E}\left\|\mathbf{K}^{-1}\left(\mathbf{u}_{2}-\mathbf{u}_{2}^{h}\right)\right\|_{0, T}+h_{E}\left\|\nabla p_{2}^{h}+\mathbf{K}^{-1} \mathbf{u}_{2}^{h}\right\|_{0, T}\right. \\
& \left.+\left\|p_{2}-p_{2}^{h}\right\|_{0, T}\right) \times\left\|p_{2}^{h} \mathbf{n}_{2} b_{E}\right\|_{0, E} .
\end{aligned}
$$

The inverse inequality (44) and inequalities $0 \leq b_{E} \leq 1, h_{E} \leq h_{T}$ lead to:

$$
h_{E}^{1 / 2}\left\|p_{2}^{h} \mathbf{n}_{2}\right\|_{0, E} \lesssim h_{T}\left\|\mathbf{K}^{-1}\left(\mathbf{u}_{2}-\mathbf{u}_{2}^{h}\right)\right\|_{0, T}+h_{T}\left\|\nabla p_{2}^{h}+\mathbf{K}^{-1} \mathbf{u}_{2}^{h}\right\|_{0, T}+\left\|p_{2}-p_{2}^{h}\right\|_{0, T} .
$$


We combine the inequalities (84) and (65), and we have (85), i.e.,

$$
\sum_{E \in \varepsilon_{h}(T) \cap_{\varepsilon_{h}}\left(\Gamma_{2}\right)} h_{E}^{1 / 2}\left\|p_{2} \mathbf{n}_{2}\right\|_{0, E} \lesssim \sum_{k \in W_{2}^{T}} h_{k}\left\|\mathbf{K}^{-1}\left(\mathbf{u}_{2}-\mathbf{u}_{2}^{h}\right)\right\|\left\|_{0, k}+\right\| p_{2}-p_{2}^{h} \|_{0, W_{2}^{T}} .
$$

Thus, the Proposition 3.2 is proved.

Theorem 3.2 (Global lower error bound). The following estimation holds:

$$
\Theta \lesssim\left\|\boldsymbol{u}-\boldsymbol{u}^{h}\right\|_{H}+\left\|p-p^{h}\right\|_{M}+\left\|\lambda-\lambda^{h}\right\|_{H^{1 / 2}\left(\Gamma_{I}\right)}+\zeta
$$

where $\zeta$ is defined by (33).

Proof. Follows directly from the Proposition 3.1 and the Proposition 3.2 .

\section{Corollary 3.1 (Main result).}

$$
\frac{\mid \text { Error }-\Theta \mid}{\zeta}=\mathcal{O}(1)
$$

where

$$
\text { Error }:=\left\|\boldsymbol{u}-\boldsymbol{u}^{h}\right\|_{H}+\left\|p-p^{h}\right\|+\left\|\lambda-\lambda^{h}\right\|_{H^{1 / 2}\left(\Gamma_{I}\right)} .
$$

\section{Conclusion and Further Works}

In this paper, we have proposed and rigorously analyzed a new a posteriori residual type error estimators for the Stokes-Darcy coupled problem on isotropic meshes. Our investigations cover conforming discretization in $2 \mathrm{D}$ and $3 \mathrm{D}$ domains. The residual type a posteriori error estimator is provided. It is proven that the a posteriori error estimate provided in this paper is both reliable and efficient. There are many issues to be addressed in this area such as the other types of a posteriori error estimates, extend the residual error estimator methods to anisotropic meshes [34] and related implementation of the adaptive finite element methods. 


\section{Acknowledgement}

The author thanks the anonymous referees for their helpful comments that significantly improve the presentation of the paper.

\section{References}

[1] W. J. Layton, F. Schieweck and I. Yotov, Coupling fluid flow with porous media flow, SIAM Journal on Numerical Analysis 40(6) (2002), 2195-2218.

DOI: https://doi.org/10.1137/S0036142901392766

[2] Ph. Clément, Approximation by finite element functions using local regularization, ESAIM: Mathematical Modelling and Numerical Analysis 9(R2) (1975), 77-84.

DOI: https://doi.org/10.1051/m2an/197509R200771

[3] R. Verfurth, A Review of a Posteriori Error Estimation and Adaptive MeshRefinement Techniques, Wiley-Teubner, Chrichester, UK, 1996.

[4] R. Verfurth, A posteriori error estimation and adaptive mesh-refinement techniques, Journal of Computational and Applied Mathematics 50(1-3) (1994), 67-83.

DOI: https://doi.org/10.1016/0377-0427(94)90290-9

[5] Alexandre Ern, Aide-Memoire Eléments Finis, 2005.

[6] W. Chen, P. Chen, M. Gunzburger and N. Yan, Superconvergence analysis of FEMs for the Stokes-Darcy system, Mathematical Methods in the Applied Sciences 33(13) (2010), 1605-1617.

DOI: https://doi.org/10.1002/mma.1279

[7] A. Mikelic and W. Jäger, On the interface boundary condition of beavers, Joseph, and Saffman, SIAM Journal on Applied Mathematics 60(4) (2000), 1111-1127.

DOI: https://doi.org/10.1137/S003613999833678X

[8] K. A. Mardal, X. C. Tai and R. Winther, A robust finite element method for DarcyStokes flow, SIAM Journal on Numerical Analysis 40(5) (2002), 1605-1631.

DOI: https://doi.org/10.1137/S0036142901383910

[9] M. Mu and J. Xu, A two-grid method of a mixed Stokes-Darcy model for coupling fluid flow with porous media flow, SIAM Journal on Numerical Analysis 45(5) (2007), 1801-1813.

DOI: https://doi.org/10.1137/050637820

[10] M. Bieterman and I. Babuska, The finite element method for parabolic equations I: A posteriori error estimation, Numerische Mathematik 40(3) (1982), 339-371.

DOI: https://doi.org/10.1007/BF01396451 
[11] I. Babuska and G. N. Gatica, A residual-based a posteriori error estimator for the Stokes-Darcy coupled problem, SIAM Journal on Numerical Analysis 48(2) (2010), 498-523.

DOI: https://doi.org/10.1137/080727646

[12] W. Liu and N. Yan, A posteriori error estimates for the control problems governed by Stokes equations, SIAM Journal on Numerical Analysis 40(5) (2002), 1850-1869.

DOI: https://doi.org/10.1137/S0036142901384009

[13] V. Girault and P. A. Raviart, Finite Element Methods for Navier-Stokes Equations: Theory and Algorithms, Springer: Berlin, 1986.

[14] Z. Chen, Finite Element Methods and their Applications, Springer: Berlin, Heidelberg, 2005.

[15] S. C. Brenner and L. R. Scott, The Mathematical Theory of Finite Element Methods, Texts in Applied Mathematics, Volume 15, Springer: Berlin, 1994.

DOI: https://doi.org/10.1007/978-1-4757-4338-8

[16] L. R. Scott and S. Zhang, Finite element interpolation of nonsmooth functions satisfying boundary conditions, Mathematics of Computation 54(190) (1990), 483-493.

DOI: https://doi.org/10.1090/S0025-5718-1990-1011446-7

[17] M. Ainsworth and J. T. Oden, A Posteriori Error Estimation in Finite Element Analysis, John Wiley and Sons: New York, ISBN 0-471-29411-X, 2000.

[18] R. Verfürth, A Posteriori Error Estimation and Adaptive Mesh Refinement Techniques, Wiley-Teubner, Chrichester, 1996.

[19] C. Carstensen, A posteriori error estimate for the mixed finite element method, Mathematics of Computation 66(218) (1997), 465-476.

DOI: https://doi.org/10.1090/S0025-5718-97-00837-5

[20] C. Carstensen and G. Dolzmann, A posteriori error estimates for mixed FEM in elasticity, Numerische Mathematik 81(2) (1998), 187-209.

DOI: https://doi.org/10.1007/s002110050389

[21] G. N. Gatica, A note on the efficiency of residual-based a posteriori error estimators for some mixed finite element methods, Electronic Transactions on Numerical Analysis 17 (2004), 218-233.

[22] J. Galvis and M. Sarkis, Non-matching mortar discretization analysis for the coupling Stokes-Darcy equations, Electronic Transactions on Numerical Analysis 26 (2007), 350-384.

[23] D. N. Arnold, F. Brezzi and M. Fortin, A stable finite element for the Stokes equations, Calcolo 21(4) (1984), 337-344.

DOI: https://doi.org/10.1007/BF02576171 
[24] M. Crouzeix and P. A. Raviart, Conforming and nonconforming finite element methods for solving the stationary stokes equations: 1, ESAIM: Mathematical Modelling and Numerical Analysis 7(3) (1973), 33-75.

[25] F. Brezzi, J. Douglas Jr., M. Fortin and L. D. Marini, Efficient rectangular mixed finite elements in two and three variables, ESAIM: Mathematical Modelling and Numerical Analysis 21(4) (1987), 581-604.

[26] C. Taylor and P. Hood, A numerical solution of the Navier-Stokes equations using the finite element technique, Computers \& Fluids 1(1) (1973), 73-100.

DOI: https://doi.org/10.1016/0045-7930(73)90027-3

[27] F. Brezzi and M. Fortin, Mixed and Hybrid Finite Element Methods, SpringerVerlag, New York, 1991.

DOI: https://doi.org/10.1007/978-1-4612-3172-1

[28] J. C. Nedelec, Mixed finite elements in $\mathbb{R}^{3}$, Numerische Mathematik 35(3) (1980), 315-341.

DOI: https://doi.org/10.1007/BF01396415

[29] P. A. Raviart and J. M. Thomas, A mixed finite element method for 2nd order elliptic problems, in Mathematical Aspects of the Finite Element Method, Lecture Notes in Mathematics, 606, Springer-Verlag, New York, 1977, pp. 292-315.

DOI: https://doi.org/10.1007/BFb0064470

[30] F. Brezzi, J. Douglas Jr. and L. D. Marini, Two families of mixed elements for second order elliptic problems, Numerische Mathematik 47(2) (1985), 217-235.

DOI: https://doi.org/10.1007/BF01389710

[31] F. Brezzi, J. Douglas Jr., R. Duran and M. Fortin, Mixed finite elements for second order elliptic problems in three variables, Numerische Mathematik 51(2) (1987), 237-250.

DOI: https://doi.org/10.1007/BF01396752

[32] Z. Chen and J. Douglas Jr., Prismatic mixed finite elements for second order elliptic problems, Calcolo 26(2-4) (1989), 135-148.

DOI: https://doi.org/10.1007/BF02575725

[33] C. Bernardi and G. Raugel, Analysis of some finite elements for the Stokes problem, Mathematics of Computation 44(169) (1985), 71-79.

DOI: https://doi.org/10.1090/S0025-5718-1985-0771031-7

[34] K. W. Houédanou and B. Ahounou, A posteriori error estimation for the StokesDarcy coupled problem on anisotropic discretization, Mathematical Methods in the Applied Sciences 40(10) (2017), 3741-3774.

DOI: https://doi.org/10.1002/mma.4261 
[35] S. Nicaise, B. Ahounou and W. Houédanou, Residual-based a posteriori error estimates for a nonconforming finite element discretization of the Stokes-Darcy coupled problem: Isotropic discretization, Afrika Matematika 27(3-4) (2016), 701-729.

DOI: https://doi.org/10.1007/s13370-015-0370-3

[36] K. W. Houédanou, J. Adetola and B. Ahounou. Residual-based a posteriori error estimates for a conforming finite element discretization of the Navier-Stokes/Darcy coupled problem, Journal of Pure and Applied Mathematics: Advances and Applications 18(1) (2017), 37-73.

DOI: http://dx.doi.org/10.18642/jpamaa_7100121867 